\title{
Properties of young contrails - a parametrisation based on large-eddy simulations
}

\author{
Simon Unterstrasser \\ Deutsches Zentrum für Luft- und Raumfahrt (DLR), Institut für Physik der Atmosphäre, Oberpfaffenhofen, \\ 82234 Wessling, Germany \\ Correspondence to: S. Unterstrasser (simon.unterstrasser@dlr.de)
}

Received: 7 August 2015 - Published in Atmos. Chem. Phys. Discuss.: 27 October 2015

Revised: 7 January 2016 - Accepted: 25 January 2016 - Published: 24 February 2016

\begin{abstract}
Contrail-cirrus is probably the largest climate forcing from aviation. The evolution of contrail-cirrus and its radiative impact depends not only on a multitude of atmospheric parameters, but also on the geometric and microphysical properties of the young contrails evolving into contrail-cirrus. The early evolution of contrails $(t<5 \mathrm{~min})$ is dominated by an interplay of ice microphysics and wake vortex dynamics. Young contrails may undergo a fast vertical expansion due to a descent of the wake vortices and may lose a substantial fraction of their ice crystals due to adiabatic heating. The geometric depth $H$ and total ice crystal number $N$ of young contrails are highly variable and depend on many environmental and aircraft parameters. Both properties, $H$ and $N$, affect the later properties of the evolving contrail-cirrus, as they control the extent of shear-induced spreading and sedimentation losses. In this study, we provide parametrisations of $H$ and $N$ after 5 min taking into account the effects of temperature, relative humidity, thermal stratification and aircraft type (mass, wing span, fuel burn). The parametrisations rely on a large data set of recent large-eddy simulations of young contrails. They are suited to be incorporated in larger-scale models in order to refine the presentday contrail initialisations by considering the processes that strongly affect the contrail evolution during the vortex phase.
\end{abstract}

\section{Introduction}

\subsection{Motivation}

Contrail-cirrus is probably the largest contribution from aviation to climate change in terms of radiative forcing
(Burkhardt and Kärcher, 2011). However, its quantification is associated with large uncertainties and the confidence of those estimates is still rated low (Boucher et al., 2013). Contrail radiative forcing is estimated by global circulation models (GCMs), whose parametrisations of contrails have been improved in the recent past. In particular, the analysis methods switched from diagnostic approaches for young (lineshaped) contrails (Ponater et al., 2002; Rap et al., 2010; Chen and Gettelman, 2013) towards a process-based treatment of contrail cirrus evolution (Burkhardt and Kärcher, 2009; Schumann, 2012).

Contrail microphysical, geometric and optical properties depend on a multitude of meteorological and aircraft parameters as investigated by high-resolution simulations (Lewellen, 2014; Unterstrasser and Görsch, 2014). In the first few minutes of a contrail's lifetime, the contrail evolution is strongly affected by the downward-propagating wake vortex pair. On the one hand, this can lead to a substantial increase in contrail depth. On the other hand, adiabatic heating may result in strong crystal loss. Both processes have an impact on the evolution of the contrail-cirrus while it spreads by vertical wind shear and produces a fall streak by sedimentation.

The present study develops and provides a parametrisation for the depth and crystal number of young contrails, which accounts for the important physics and considers the dominant parameters, namely relative humidity, temperature, thermal stratification and aircraft parameters. The parametrisation is based on the evaluation of a large data set of recent large-eddy simulations (LESs). Due to its simple analytic form, it is suited to be incorporated into large-scale models where it can replace present-day contrail initialisation with ad hoc assumptions, where vortex phase processes are only 
very roughly captured. This approach exhibits a way to condense information gained from LESs such that it finds its way into global-scale models.

The presented parametrisation covers a much larger parameter space and is more universal than the parametrisation given by Unterstrasser et al. (2008) and extended by Kärcher et al. (2009). Clearly, the present work updates the earlier versions.

In the recent past, the effect of biofuels on contrail properties aroused scientific interest. In particular, the likely reduction in the initial ice crystal number can have a significant effect, even though the initial differences in ice crystal number are reduced during the vortex phase (Lewellen, 2014; Unterstrasser, 2014). The effect of biofuels may be overestimated, if crystal loss is neglected. The presented parametrisation accounts for the crystal loss; hence, it avoids this problem.

Moreover, the present approach complements a parametrisation describing the long-term contrail-cirrus evolution in Lewellen (2014) where the ice crystal number is an important input parameter.

\subsection{Contrail microphysics and dynamics}

Since the design of the proposed parametrisations is motivated by the physical processes involved in contrail evolution, this section will give a short overview of various aspects of the contrail evolution. In the remainder of the text, we assume that the environment is sufficiently cold and moist, such that contrails form (Schumann, 1996) and persist (i.e. that the air is supersaturated). In general, contrail development is divided into three temporal phases based upon the governing physical processes: the jet, vortex and dispersion phase (Hoshizaki et al., 1975).

The jet phase denotes the first several seconds, in which the lift generating airflow over the wings transforms into a counter-rotating vortex pair. The hot exhaust jets mix with the cold ambient air, expand and get entrained into the forming vortices (Paoli et al., 2013). Formation of ice crystals is completed within $1 \mathrm{~s}$ behind the aircraft and their number $N_{\text {form }}$ depends inter alia on the number of emitted soot particles $N_{\text {soot }}$ (Kärcher and Yu, 2009). Both quantities are usually given in units, "number per metre (of flight path)".

In the vortex phase, which lasts several minutes, the counter-rotating vortices propagate downward up to several hundreds of metres. Whereas most of the exhaust (including the ice crystals) is carried down in the vortex system (called primary wake), some of it gets detrained and forms a curtain between the cruise altitude and the actual vortex position (called secondary wake). After vortex break-up, buoyancy may cause a major fraction of the exhaust to rise back to the original emission altitude (e.g. Unterstrasser et al., 2014). Ice crystals sublimate and get lost due to adiabatic heating in the primary wake (Sussmann and Gierens, 1999). The number of ice crystals surviving the vortex phase, $N$, is highly variable and depends on a multitude of environmental condi- tions and aircraft properties (Lewellen et al., 2014; Lewellen, 2014; Unterstrasser, 2014; Unterstrasser and Görsch, 2014). Ice crystals in the secondary wake face the fresh supersaturated air and grow, such that they often contain most of the contrail ice mass at the end of the vortex phase.

The dispersion phase treats the contrail-to-cirrus transition and starts once the vortices have lost their coherent structure and vorticity has reached ambient levels. Not only the environmental conditions during this transition are important, but also the early contrail properties matter for the later contrail-cirrus properties (Unterstrasser and Gierens, 2010a, b; Lewellen, 2014; Unterstrasser, 2014; Unterstrasser and Görsch, 2014). More specifically, Unterstrasser and Görsch (2014) investigated the impact of aircraft type on contrailcirrus evolution; they found that contrails differed a lot in terms of vertical extent and ice crystal number after the vortex phase and simulation tests showed that both the contrail depth and ice crystal number after the vortex phase are relevant for the later properties of the contrail-cirrus.

Compared to the timescales of natural processes like vertical turbulent mixing, the initial expansion during the vortex phase can be viewed as a sudden event. The extent of the shear-induced horizontal spreading scales linearly with the contrail depth. Later, the depth of contrail-cirrus will increase by sedimentation and the formation of a fall streak given that the supersaturated layer is sufficiently deep. Moreover, radiative lifting may increase the contrail vertical extent (Lewellen, 2014; Unterstrasser and Gierens, 2010b). The effectiveness of sedimentation depends on the ice crystal size distribution. In a simplified picture (where we think of a monodispersed size distribution of the ice crystals), the size is determined by contrail ice mass and ice crystal number. As the ice mass of the emerging contrail-cirrus is mainly controlled by the supply of ambient water vapour, contrail cirrus properties are more susceptible to changes of number than mass of the young contrail (Unterstrasser and Gierens, $2010 b)$. Thus, our study focuses on ice crystal number $N$ rather than ice crystal mass $I$.

The number of ice crystals after the vortex phase $N$ is given by

$N_{\text {surv }}=N_{\text {form }} \times f_{\mathrm{Ns}}$,

where $f_{\mathrm{Ns}}$ is the fraction of ice crystals surviving the vortex phase (survival fraction). $N_{\text {form }}$ is the number of generated ice crystals in the beginning and is given by

$N_{\text {form }}=\frac{\dot{m}_{\mathrm{f}}}{U} \mathrm{EI}_{\text {iceno }}$,

where $\mathrm{EI}_{\text {iceno }}$ is the "emission" index of ice crystals. In sootrich regimes, homogeneous freezing of water-activated soot is the primary ice formation mechanism (Kärcher and $\mathrm{Yu}$, 2009 ) and $\mathrm{EI}_{\text {iceno }}$ may be given by $f_{\mathrm{A}} \times \mathrm{EI}_{\text {soot }}$, where $f_{\mathrm{A}}$ is the fraction of activated soot particles. A schematic overview is given in Fig. 1. Evaluation of $f_{\mathrm{A}}$ and the corresponding 


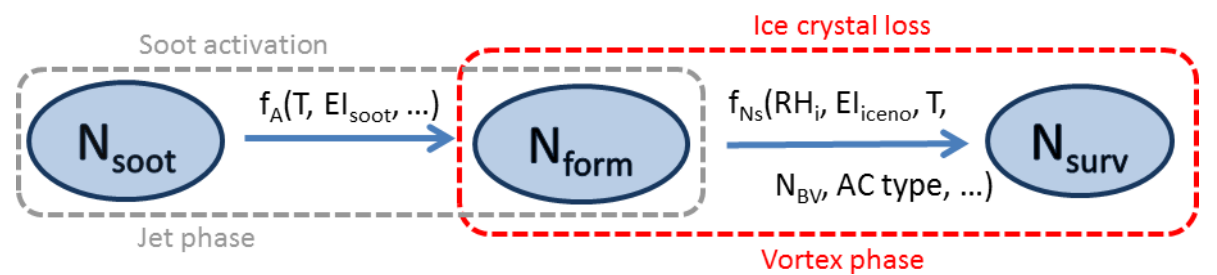

Figure 1. From soot emission to contrail ice crystal number: number of emitted soot particles $N_{\text {soot }}$, generated ice crystals $N_{\text {form }}$ and ice crystals $N_{\text {surv }}$ present after vortex break-up and relevant for contrail-to-cirrus transition. $f_{\mathrm{A}}$ denotes the fraction of activated soot particles (during the first seconds behind the aircraft), and $f_{\mathrm{Ns}}$ the fraction of ice crystals surviving the adiabatic heating during the vortex phase (during the first $\sim 5 \mathrm{~min}$ ). The displayed quantities have units per metre (of flight path), which can be converted into emission indices following Eq. (2) and analogous expressions. The present study focuses on the loss process during the vortex phase (red box). For soot-poor

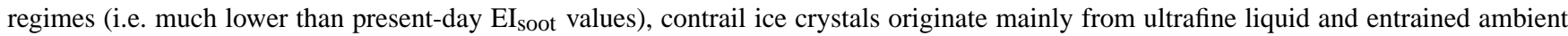
particles and the simple picture expressed by $N_{\text {form }}=f_{\mathrm{A}} \times N_{\text {soot }}$ in the grey box is not valid any longer (Kärcher and Yu, 2009).

soot emission index $\mathrm{EI}_{\text {soot }}$ is left to others (Kärcher et al., 1998; Kärcher and Yu, 2009). In this paper, we simply vary $\mathrm{EI}_{\text {iceno }}$ and parametrise $f_{\mathrm{Ns}}$.

Section 2 describes the data set of simulations and explains the vortex phase processes in more detail. Section 3 presents analytical parametrisations of the survival fraction, the depth and the width of $5 \mathrm{~min}$ old contrails. Section 4 discusses the relevance of the input parameters and the effects of a soot reduction. Section 5 discusses the robustness of the presented parametrisations and conclusions are drawn in Sect. 6. The Appendix A includes instructions on how to implement the parametrisation and the Supplement contains a FORTRAN source code file of the parametrisation. Throughout the paper, parametrisations and fit functions are identified by a " " symbol, e.g. $\hat{f}_{\mathrm{Ns}}$ or $\hat{N}_{\text {surv }}$.

\section{Data set of large-eddy simulations}

The parametrisation is based on 3-D LES simulations of the contrail vortex phase with EULAG-LCM (Sölch and Kärcher, 2010) that are described in two recent publications (Unterstrasser, 2014; Unterstrasser and Görsch, 2014). Ad-

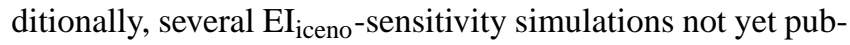
lished are considered.

The simulations start at wake age of several seconds. Then it is reasonable to assume ice crystal nucleation and wake vortex roll-up to be finished. A pair of counter-rotating Lamb Oseen vortices with specified circulation $\Gamma_{0}$ is prescribed together with two circular plumes containing $N_{\text {form }}$ ice crystals. A more detailed description of the simulation set-up is given in the aforementioned references.

A list of all 81 simulations is given in Table A2. Columns 2-7 summarise the parameters of the simulations that are incorporated into the parametrisation:

- temperature at cruise altitude $T_{\mathrm{CA}}$

- ambient relative humidity $\mathrm{RH}_{\mathrm{i}}$ or supersaturation

$$
s_{\mathrm{i}}=\mathrm{RH}_{\mathrm{i}}-1
$$

$$
\begin{aligned}
& \text { - Brunt-Väisälä frequency } N_{\mathrm{BV}} \\
& \text { - aircraft (AC) type } \\
& \text { - water vapour emission } \\
& \text { - ice crystal "emission” index } \mathrm{EI}_{\text {iceno }} \text {. }
\end{aligned}
$$

The data set covers a large parameter space: $T_{\mathrm{CA}}$ ranges between 209 and $225 \mathrm{~K}$ : the temperature region, where the majority of contrail-producing flights occur. $\mathrm{RH}_{\mathrm{i}}$ ranges between 100 and $140 \%$ and represents conditions where contrail persistence is likely. $N_{\mathrm{BV}}$ values of 0.005 and $0.0115 \mathrm{~s}^{-1}$ represent typical upper tropospheric stable con-

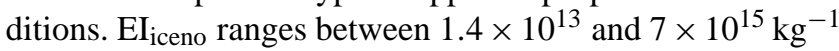
and covers values of the present-day fleet as well as of future engines with potentially lower soot emissions.

Variations of $T_{\mathrm{CA}}, \mathrm{RH}_{\mathrm{i}}, \mathrm{EI}_{\mathrm{iceno}}$ and fuel flow have a direct impact on contrail ice microphysics and do not affect the wake vortex evolution as latent heating effects are negligible. Those variations have been examined in Unterstrasser (2014) (from now on referred to as U2014) for a contrail generated by an aircraft of type B777/A340. Variations of $N_{\mathrm{BV}}$ and AC type mainly affect the wake vortex evolution and have been examined in Unterstrasser and Görsch (2014) (from now on referred to as UG2014).

We will shortly describe a few parameter studies selected from these two publications. This should illustrate the basic microphysical and dynamical mechanisms, exemplify the impact of the above-mentioned parameters and point out the processes leading to the variability in $H$ and $N_{\text {surv }}$.

Figure 2 shows vertical profiles of ice crystal number after 5-6 min; i.e. at a time the vortices have already broken up and the contrail reached its full vertical extent (for now neglecting the later formation of a fall streak). Such plots have been used to determine the final vertical displacement $z_{\text {desc }}$ of the wake vortex and the contrail depth $H$. Having in mind likely future applications of the parametrisations in large-scale models, where physics of the contrail-cirrus transition are anyway more simplified than in LES approaches, 

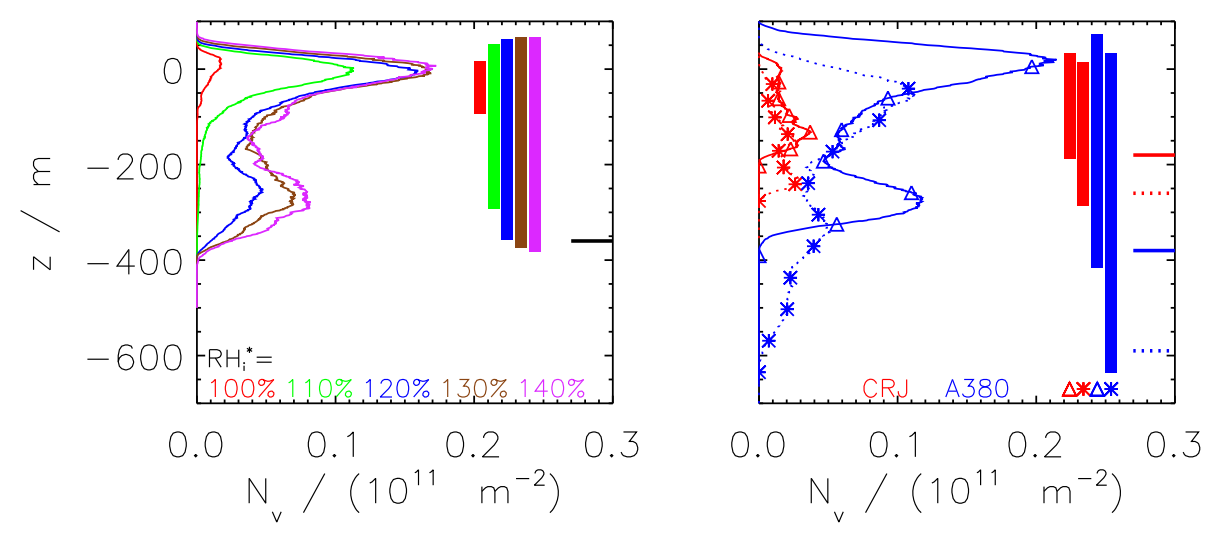

Figure 2. Vertical profiles of contrail ice crystal number after $t=5-6 \mathrm{~min}$ (well after vortex break-up) are shown. The horizontal bars indicate the value of $z_{\text {desc }}$. The rectangles illustrate the vertical extent of the contrail and their heights are equal to the corresponding contrail depths $H$. Left panel: variation of relative humidity (see legend) for a B777-type aircraft and $N_{\mathrm{BV}}=0.0115 \mathrm{~s}^{-1}$. Right panel: variation of aircraft type (see legend) and stability (solid with triangles: $N_{\mathrm{BV}}=0.0115 \mathrm{~s}^{-1}$, dotted with stars: $N_{\mathrm{BV}}=0.005 \mathrm{~s}^{-1}$ ) for RH $\mathrm{R}_{\mathrm{i}}=140 \%$. In all cases $T=217 \mathrm{~K}$. The cruise altitude of the contrail generating aircraft is at $z=0$.
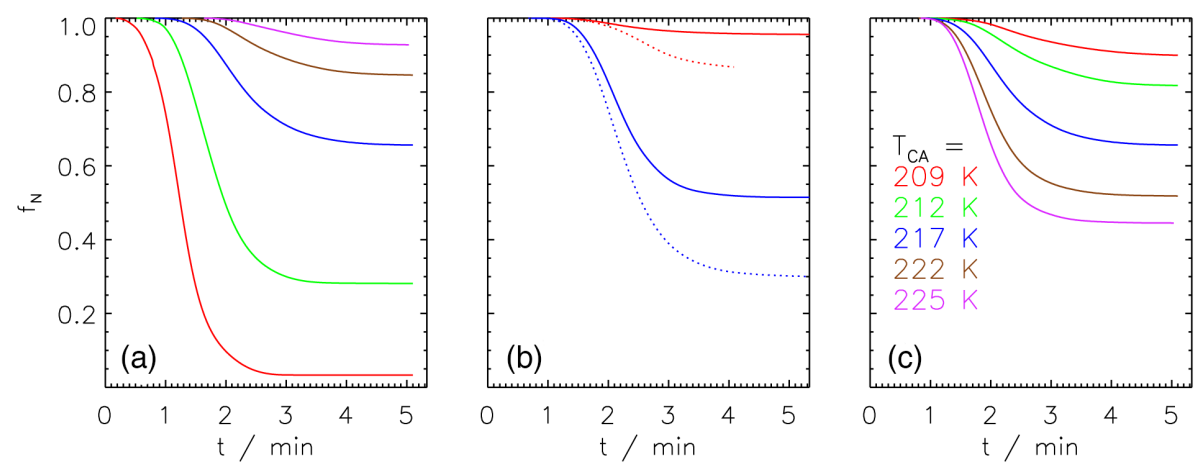

Figure 3. Temporal evolution of normalised ice crystal number is shown. Panels (a) and (b) show the same simulations as Fig. 2, except that in panel (b) $\mathrm{RH}_{\mathrm{i}}=120 \%$ instead of $140 \%$. Panel (c) shows the effect of a temperature variation (see legend) at $\mathrm{RH}_{\mathrm{i}}=120 \%$ and $N_{\mathrm{BV}}=0.0115 \mathrm{~s}^{-1}$.

uncertainties of $\pm 25 \mathrm{~m}$ in the $H$ determination are certainly acceptable.

The left panel shows the effect of a $\mathrm{RH}_{\mathrm{i}}$ variation. The evolution of the wake vortex pair is unaffected by such a variation. Hence, the final vertical displacement $z_{\text {desc }}$ (marked by the black bar) is basically identical. In this specific case, ice crystal loss either reduces the contrail depth $H\left(\mathrm{RH}_{\mathrm{i}} \leq\right.$ $110 \%)$ or the crystal abundance in the primary wake $\left(\mathrm{RH}_{\mathrm{i}}=\right.$ $120 \%$ ). Thus, contrail depth $H$ depends on $\mathrm{RH}_{\mathrm{i}}$ and the boxes show our choices of $H$ values.

Due to adiabatic heating in the primary wake, sublimation and loss of ice crystals occurs even in a supersaturated environment. Figure 3 a shows the normalised ice crystal number $f_{\mathrm{N}}(t)=N(t) / N_{\text {form }}$ over time. Once the vortex descent stops after a few minutes, the rapid crystal loss ceases and the $f_{\mathrm{N}}$ value at the end of the simulation defines the survival fraction $f_{\mathrm{Ns}}$. For the given $\mathrm{RH}_{\mathrm{i}}$ variation, $f_{\mathrm{Ns}}$ ranges from less than $5 \%$ to more than $90 \%$.
As a next step, contrails of six different aircraft types were investigated in UG2014. This ranged from a regional airliner Bombardier CRJ to the largest passenger aircraft Airbus A380. The wing span, mass, speed and fuel flow of the aircraft determines the circulation and the separation of the wake vortices, the water vapour emission and the number of ice crystals (for a given $\mathrm{EI}_{\text {iceno). The specific aircraft- }}$ dependent choices in UG2014 rely on BADA (Base of Aircraft Data) estimates (Nuic, 2011). Table 1 lists the most relevant aircraft-dependent properties. More details are listed in Table 1 of UG2014.

The right panel of Fig. 2 illustrates the impact of AC type and thermal stratification on the wake vortex evolution. For the displayed $\mathrm{RH}_{\mathrm{i}}=140 \%$ simulations, crystal loss is low (not shown) and the contrail profiles reveal the final vertical displacement $z_{\text {desc }}$ of the vortices. $z_{\text {desc }}$ is larger for larger aircraft and weaker stratification. The trends in contrail depth are the same. Note that for stronger stratification, buoyancy effects are stronger and more ice crystals are pushed back 
Table 1. List of aircraft-dependent parameters. Columns 2-7: values used in UG2014; columns 8-10: values used in Naiman et al. (2011); column 11: values used in Paugam et al. (2010); column 12: values used in Picot et al. (2015).

\begin{tabular}{|c|c|c|c|c|c|c|c|c|c|c|c|}
\hline Aircraft type & CRJ & $\begin{array}{l}\text { A320 } \\
\text { B737 }\end{array}$ & $\begin{array}{l}\text { A300 } \\
\text { B767 }\end{array}$ & $\begin{array}{l}\text { A350 } \\
\text { B777 }\end{array}$ & B747 & A 380 & B737N & B767N & B747N & B747P1 & B747P2 \\
\hline Wing span $b / \mathrm{m}$ & 21.2 & 34.4 & 47.6 & 60.9 & 64.4 & 79.8 & 34.3 & 47.2 & 64.5 & 59.9 & 59.9 \\
\hline Circulation $\Gamma_{0} /\left(\mathrm{m}^{2} \mathrm{~s}^{-1}\right)$ & 130 & 240 & 390 & 520 & 590 & 720 & 246 & 391 & 646 & 600 & 565 \\
\hline Water vapour emission $\mathcal{V} /\left(\mathrm{g} \mathrm{m}^{-1}\right)$ & 1.77 & 3.70 & 7.26 & 15.0 & 13.8 & 20.0 & 3.13 & 7.25 & 14.5 & 12.5 & 15.0 \\
\hline Descent speed $w_{0} /\left(\mathrm{m} \mathrm{s}^{-1}\right)$ & 1.24 & 1.41 & 1.66 & 1.73 & 1.85 & 1.83 & 1.45 & 1.68 & 2.03 & 2.03 & 1.91 \\
\hline Vortex timescale $t_{0} / \mathrm{s}$ & 13.4 & 19.1 & 22.5 & 27.6 & 27.3 & 34.3 & 18.5 & 22.0 & 24.9 & 23.1 & 24.6 \\
\hline
\end{tabular}

to cruise altitude (CA). For the A380 aircraft, these restoring forces even lead to an overshooting and the contrail may reach altitudes nearly $100 \mathrm{~m}$ above the CA.

The different wake vortex evolutions have consequences on the crystal loss extent and more crystals are lost for larger $z_{\text {desc }}$ as adiabatic heating is stronger. Thus, fewer ice crystals are lost in a more stable environment and for smaller aircraft as Fig. 3b demonstrates for $\mathrm{RH}_{\mathrm{i}}=120 \%$ cases. Nevertheless, a A380 contrail contains more ice crystals than a CRJ contrail, as the fuel flow is higher and thus $N_{\text {form }}$ is larger assuming an aircraft-independent value for $\mathrm{EI}_{\text {iceno. }}$.

Finally, the effect of an $\mathrm{EI}_{\text {iceno }}$ variation is discussed. Figure 4 shows the temporal evolution of ice crystal mass (top) and number (middle) for various $\mathrm{EI}_{\mathrm{iceno}}$ values. The higher $\mathrm{EI}_{\text {iceno }}$ and $N_{\text {form }}$ are, the smaller is the mean mass of the ice crystals. Thus, a larger fraction of them is lost. There is a subtle difference in the way $\mathrm{EI}_{\text {iceno }}$ affects $f_{\mathrm{N}}$ compared to $\mathrm{RH}_{\mathrm{i}}$ or $T_{\mathrm{CA}}$ and will later be reflected in the design of the parametrisation. Variation of the latter two variables affects the ice mass evolution (see Fig. 3 in U2014), which then has implications on the number evolution. For a $\mathrm{EI}_{\text {iceno }}$ variation, on the other hand, the ice mass evolution is basically identical. Note that the differences after 2 min are mostly due to different growth of detrained crystals in the secondary wake, which is irrelevant for the number loss in the primary wake. This has also implications on the contrail depth. Figure 4 (bottom) shows vertical profiles of ice crystal mass. The contrail depth is unaffected by a variation of $\mathrm{EI}_{\text {iceno }}$ and by the implied change in crystal loss.

In Sect. 3.3 of U2014, the width $r_{\mathrm{SD}}$ of the initial ice crystal size distribution was varied and the effect of an $r_{\mathrm{SD}}$ variation is similar to that of an $\mathrm{EI}_{\text {iceno variation; i.e. the mass }}$ budget and the contrail depth are unaffected, but the extent of crystal loss changes. This suggests that contrail depth is only affected by parameter variations that change the mass evolution or the wake vortex evolution.

\section{Parametrisation}

The examples in the preceding section highlighted some basic mechanisms. This section provides the theoretical background to better understand the observed sensitivities and
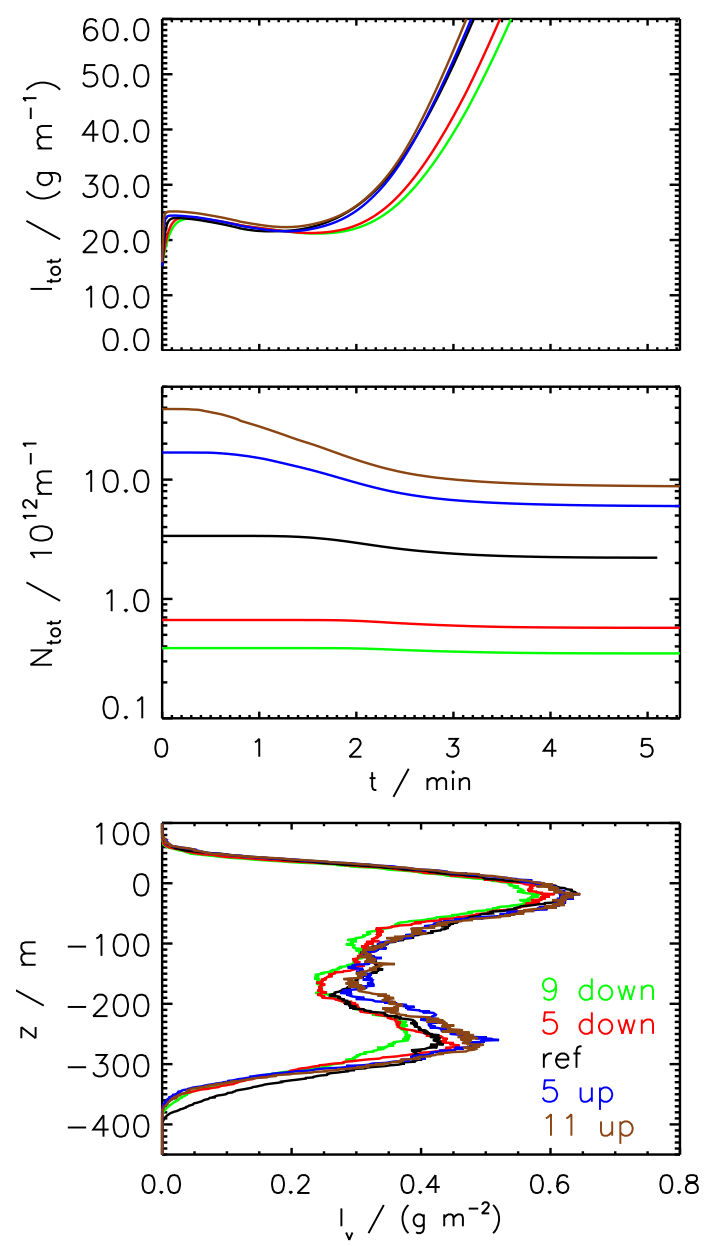

Figure 4. Variation of $\mathrm{EI}_{\mathrm{iceno}}$ for a B777/A350-type aircraft, $\mathrm{RH}_{\mathrm{i}}=$ $120 \%, T=217 \mathrm{~K}$ and $N_{\mathrm{BV}}=0.0115 \mathrm{~s}^{-1}$. The reference simulation (ref) uses $\mathrm{EI}_{\text {iceno, ref }}=2.8 \times 10^{14} \mathrm{~kg}^{-1}$. In further simulations $\mathrm{EI}_{\text {iceno }}$ is lower or higher (see legend for the scaling factors). Temporal evolution of total ice crystal mass (top) and number (middle) and vertical profiles of ice crystal mass after 5 min (bottom).

allows for the development of process-based parametrisations for the survival fraction and the contrail depth. For the contrail-to-cirrus transition the ice crystal number matters, which is the product of initial ice crystal number and survival fraction. 


\subsection{Characteristic length scales}

We introduce three length scales that help to understand and explain the observed processes in a young contrail. A similar attempt has already been undertaken in Unterstrasser et al. (2008), where three timescales were used to better explain the observed processes in the downward sinking contrail. The three length scales are

$-z_{\text {desc }}$, which measures the final vertical displacement of the wake vortex;

- $z_{\text {atm, }}$ which measures the effect of the ambient supersaturation on the ice crystal mass budget;

- $z_{\text {emit }}$, which measures the effect of the water vapour (WV) emission on the ice crystal mass budget.

We will later see that the approximations of $f_{\mathrm{Ns}}$ and $H$ take simple forms, if expressed in terms of $z_{\Delta}$, which is a linear combination of the three length scales:

$z_{\Delta}=\alpha_{\mathrm{atm}} \times z_{\mathrm{atm}}+\alpha_{\mathrm{emit}} \times z_{\mathrm{emit}}-\alpha_{\mathrm{desc}} \times \hat{z}_{\mathrm{desc}}$

with positive weights $\alpha_{\mathrm{i}}$. The equation describes a balance between the WV surplus (raising $\mathrm{RH}_{\mathrm{i}}$ ) and wake vortexinduced adiabatic heating (lowering $\mathrm{RH}_{\mathrm{i}}$ ). Note the minus sign in front of the $\hat{z}_{\text {desc }}$ term. In the following, we will define the three length scales. Whereas $z_{\text {atm }}$ and $z_{\text {emit }}$ are analytically defined, $z_{\text {desc }}$ is determined from simulations and an analytical approximation $\hat{z}_{\text {desc }}$ enters the balance Eq. (3).

\subsubsection{Length scale $z_{\text {desc }}$}

In scenarios with $\mathrm{RH}_{\mathrm{i}}=140 \%$, contrails reach their full vertical extent, as ice crystal loss is small. The determination of $z_{\text {desc }}$ is based on visually inspecting vertical profiles of such simulations (as exemplified in Fig. 2). Following UG2014 or a shortened derivation in Sect. A2, $z_{\text {desc }}$ can be approximated by

$\hat{z}_{\text {desc }}=\sqrt{\frac{8 \Gamma_{0}}{\pi N_{\mathrm{BV}}}}$.

$\hat{z}_{\text {desc }}$ depends solely on $\Gamma_{0}$ and $N_{\mathrm{BV}} . \Gamma_{0}$ denotes the initial circulation of the wake vortices. It can be computed via Eq. (A1) or empirically determined via Eq. (A5). Compared to a variation of the aircraft type (affecting $\Gamma_{0}$ ) and the ambient stratification, variations of vertical wind shear and turbulence are of secondary importance (at least for stratification values typical of the upper troposphere); see, for example, Unterstrasser et al. (2014).

\subsubsection{Length scale $z_{\text {atm }}$}

We consider a supersaturated air parcel that we assume to be void of ice crystals; i.e. no deposition/sublimation is going on. Then we estimate the vertical displacement $z_{\text {atm }}$ of the parcel that is necessary to reduce the relative humidity to saturation. This means that the water vapour concentration of a supersaturated air parcel is equal to that of a saturated parcel at some higher temperature. Using the ideal gas law $\rho_{\mathrm{v}}=\frac{e_{\mathrm{V}}(T)}{R_{\mathrm{V}} T}$, we then have

$\left(1+s_{\mathrm{i}}\right) \frac{e_{\mathrm{S}}\left(T_{\mathrm{CA}}\right)}{T_{\mathrm{CA}}}=\frac{e_{\mathrm{s}}\left(T_{\mathrm{CA}}+\Gamma_{\mathrm{d}} z_{\mathrm{atm}}\right)}{\left(T_{\mathrm{CA}}+\Gamma_{\mathrm{d}} z_{\mathrm{atm}}\right)}$

with supersaturation $s_{\mathrm{i}}$, saturation vapour pressure $e_{\mathrm{S}}$ and dry adiabatic lapse rate $\Gamma_{\mathrm{d}}$. The non-linear equation in $z_{\mathrm{atm}}$ is solved using a simple iterative numerical approach. A similar, yet linearised, version of this definition was given in Unterstrasser et al. (2008) (there named $\delta_{\text {crit }}$ ).

Table A2 lists the values of $z_{\mathrm{atm}}$ for given $\mathrm{RH}_{\mathrm{i}}$ and $T_{\mathrm{CA}}$ and shows the dominant impact of $\mathrm{RH}_{\mathrm{i}}$.

Using the wake vortex descent speed $w, z_{\text {atm }}$ can be converted into a timescale $t_{\mathrm{atm}}$ that gives the link to the observed $\mathrm{RH}_{\mathrm{i}}$-separated onset of crystal loss in Fig. 3a.

\subsubsection{Length scale $z_{\text {emit }}$}

We consider an ice crystal-free saturated aircraft plume. Then the emitted water vapour increases the vapour concentration $\rho$ in the plume such that it is supersaturated. The water vapour emission $\mathcal{V}$ (in units kg per metre of flight path) is determined mainly by the fuel flow of the aircraft; see Eq. (A8). The additional water vapour can be seen as an additional concentration

$\rho_{\text {emit }}=\frac{\mathcal{V}}{A_{\mathrm{p}}}$

assuming a uniform distribution over a certain plume area $A_{\mathrm{p}}$. An aircraft-dependent approximation of the plume area is given in Sect. A4. Similar to the $z_{\text {atm }}$ derivation, we determine the vertical displacement $z_{\text {emit }}$ that is necessary to reduce the relative humidity to saturation.

$\frac{e_{\mathrm{s}}\left(T_{\mathrm{CA}}\right)}{R_{\mathrm{v}} T_{\mathrm{CA}}}+\rho_{\mathrm{emit}}=\frac{e_{\mathrm{S}}\left(T_{\mathrm{CA}}+\Gamma_{\mathrm{d}} z_{\mathrm{emit}}\right)}{R_{\mathrm{v}}\left(T_{\mathrm{CA}}+\Gamma_{\mathrm{d}} z_{\mathrm{emit}}\right)}$

The non-linear equation in $z_{\text {emit }}$ is solved using a simple iterative numerical approach.

Both quantities, $A_{\mathrm{p}}$ and $\mathcal{V}$, scale with $b^{2}$, such that $\rho_{\mathrm{emit}}$ and $z_{\text {emit }}$ are nearly independent of the AC type. Table A2 lists the values of $z_{\mathrm{emit}}$, which shows a strong impact of $T_{\mathrm{CA}}$ and the second-order effect of AC type.

Figure $3 c$ shows the ice crystal number evolution for various temperatures. Whereas the onset of crystal loss is similar (unlike to cases, when $\mathrm{RH}_{\mathrm{i}}$ is varied), crystal loss is faster for higher temperatures. This is simply due to the fact that the derivative $\frac{\mathrm{d}}{\mathrm{d} T} e_{\mathrm{s}}$ is higher at a higher temperature and more ice mass has to sublimate to maintain saturation.

We see in our simulations that the ice and water vapour are not homogeneously distributed over the plume. Thus, our picture of a uniform bulk $\rho_{\text {emit }}$ may be overly simple, if the 
effects of plume inhomogeneity on crystal loss do not average out.

Moreover, the plume area is only vaguely determined in our approach. We neglect, for example, the possible impact of stratification, which affects the detrainment of ice crystals out of the vortices and the entrainment of ambient air into the vortices.

On the other hand, a possible systematic underestimation of $A_{\mathrm{p}}$, for example, would result in an overestimation of $q_{\mathrm{emit}}$ and $z_{\text {emit }}$. Our parametrisation is designed in a way that such a bias can be compensated for by choosing a smaller value for weight $\alpha_{\text {emit }}$.

We will later demonstrate that the present approach is advanced enough to capture the most relevant dependencies.

\subsection{Crystal loss}

The latter two length scales were introduced assuming an ice crystal-free parcel, yet they are also meaningful for an ice crystal laden plume. In this case, the excess moisture (WV emission + ambient supersaturation) quickly deposits on the ice crystals such that relative humidity reaches saturation and the ice mass is the sum of both contributions. Ongoing heating of the plume causes a slight subsaturation and sublimation of ice mass. After a downward displacement of $z_{\mathrm{atm}}$, the ice crystals have lost as much mass as was contributed by the ambience (neglecting a small time delay); i.e. the remaining ice mass equals that of the WV emission.

The left panel of Fig. 5 depicts the simulated $f_{\mathrm{Ns}}$ values as a function of $z_{\Delta}$ for several subsets of simulations. The choice of the weights $\alpha_{\mathrm{i}}$ for the computation of $z_{\Delta}$ will be discussed later. We approximate $f_{\mathrm{Ns}}$ by an arc tangent function (grey curve), which depends solely on $z_{\Delta}$. The approximated survival fraction $\hat{f}_{\mathrm{Ns}}$ is defined by

$\hat{f}_{\mathrm{Ns}}=\hat{a}\left(z_{\Delta}\right)$,

where

$\hat{a}(x)=\beta_{0}+\frac{\beta_{1}}{\pi} \arctan \left(\alpha_{0}+(x / 100 \mathrm{~m})\right)$.

Values of $\hat{a}$ below 0 and above 1 are clipped. Again, values of the fit parameters $\beta_{0}, \beta_{1}$ and $\alpha_{0}$ are provided later.

The first row of Fig. 5 shows $f_{\mathrm{Ns}}$ for a basic $\mathrm{RH}_{\mathrm{i}}-T_{\mathrm{CA}}$ variation for one AC type. The selected simulations are listed in block 1 of Table A2 and all have the same $\hat{z}_{\text {desc }} \cdot z_{\text {atm }}$ and $z_{\text {emit }}$ vary over large ranges, as they mainly depend on $\mathrm{RH}_{\mathrm{i}}$ and $T_{\mathrm{CA}}$. Thus, this sub-panel demonstrates that the $\mathrm{RH}_{\mathrm{i}}$ and $T_{\mathrm{CA}}$ sensitivity of $f_{\mathrm{Ns}}$ is well captured by our $\hat{a}\left(z_{\Delta}\right)$ approach.

In a next step, the AC type is varied (block 2/row 2 of the table/figure). The aircraft type strongly affects the wake vortex properties, i.e. the descent speed, the time of vortex break-up and the final vertical displacement. Thus, this simulation subset focuses on the variation of $z_{\text {desc }}$ (and $z_{\text {atm }}$ ). $f_{\mathrm{Ns}}$ is smaller for larger aircraft. This trend is also captured by the approximation, as $\hat{z}_{\text {desc }}$ is larger and, correspondingly, $z_{\Delta}$ is smaller.

Row 3 of the figure extends the simulation set by cases with weaker stratification where $z_{\text {desc }}$ is larger and fewer ice crystals survive. The approximation is able to predict the behaviour, yet the distances between the data points and the approximation are larger than in row 1.

So far, the prediction of ice crystal number loss is based on a balance equation of the ice crystal mass. This is suitable and works for the simulations depicted in rows 1-3, as they all use the reference ice crystal emission index $\mathrm{EI}_{\text {iceno, ref }}=$ $2.8 \times 10^{14} \mathrm{~kg}^{-1}$. In these cases a certain ice mass change can be linked to a certain ice crystal loss. As discussed in Sect. 2, this does not hold any longer, when $\mathrm{EI}_{\text {iceno }}$ is varied. To cover the effects of a $\mathrm{EI}_{\text {iceno }}$ variation, the parametrisation must be extended. Note that, so far, $\mathrm{EI}_{\text {iceno }}$ did not enter the computations for the length scales, $z_{\Delta}$ nor for $\hat{a}$.

It is clear that $z_{\text {desc }}$ is unaffected by a $\mathrm{EI}_{\text {iceno }}$ variation and our strategy is that the terms $\alpha_{\text {atm }} \times z_{\text {atm }}$ and $\alpha_{\text {emit }} \times z_{\text {emit }}$ in the balance equation are modified. We keep the definitions of $z_{\text {atm }}$ and $z_{\text {emit }}$ and make the weights $\alpha_{\text {atm }}$ and $\alpha_{\text {emit }}$ dependent of $\mathrm{EI}_{\text {iceno. }}$.

We define the normalised emission index $\mathrm{EI}_{\text {iceno }}^{*}$ as $\mathrm{EI}_{\text {iceno }} / \mathrm{EI}_{\mathrm{iceno}}$, ref and use a correction term of type $\mathrm{EI}_{\text {iceno }}^{*}-\gamma$ for the weights. Subsequently, the weights $\alpha_{\text {atm }}$ and $\alpha_{\text {emit }}$ are smaller for higher EI iceno. This reduces $z_{\Delta}$ and, correspondingly, $\hat{f}_{\mathrm{Ns}}$ becomes smaller, as desired.

Rows 4 and 5 shows the simulated survival fractions for a small $\mathrm{EI}_{\text {iceno }}$ variation for six $\mathrm{AC}$ types and a large $\mathrm{EI}_{\text {iceno }}$ variation for the default AC type. These sub-panels demonstrate that the effect of an $\mathrm{EI}_{\text {iceno }}$ variation can be well captured by the corrected approximation. Note that without this correction the various symbols of a specific colour would all lie on one vertical line (i.e. identical $z_{\Delta}$ ).

So far, the fuel flow changed only with AC type. As a last test, we vary the fuel flow (i.e. the WV emission $\mathcal{V}$ ) for a fixed AC type. The sensitivity simulations are listed in block 5 in Table A2 (originally discussed in Fig. 9 of U2014). Figure 12 nicely reveals that the sensitivity to $\mathcal{V}$ is well represented in the parametrisation; for $\mathrm{RH}_{\mathrm{i}}=110 \%$ the agreement is excellent, for $\mathrm{RH}_{\mathrm{i}}=120 \%$ it is reasonable.

In the left panels of Fig. 5 the vertical distance of the symbol $\left(f_{\mathrm{Ns}}\right)$ to the grey curve $(\hat{f})$ shows the absolute error $f_{\mathrm{abs}}=\hat{f}_{\mathrm{Ns}}-f_{\mathrm{Ns}}$. In the right panels, scatter plots of $\hat{f}_{\mathrm{Ns}}$ vs. $f_{\mathrm{Ns}}$ are depicted and the errors can be more conveniently assessed. Furthermore, the root mean square of the absolute errors are given for each subset of simulations. The absolute errors are mostly below 0.1 for each subset, which proves the suitability of the proposed parametrisation. 

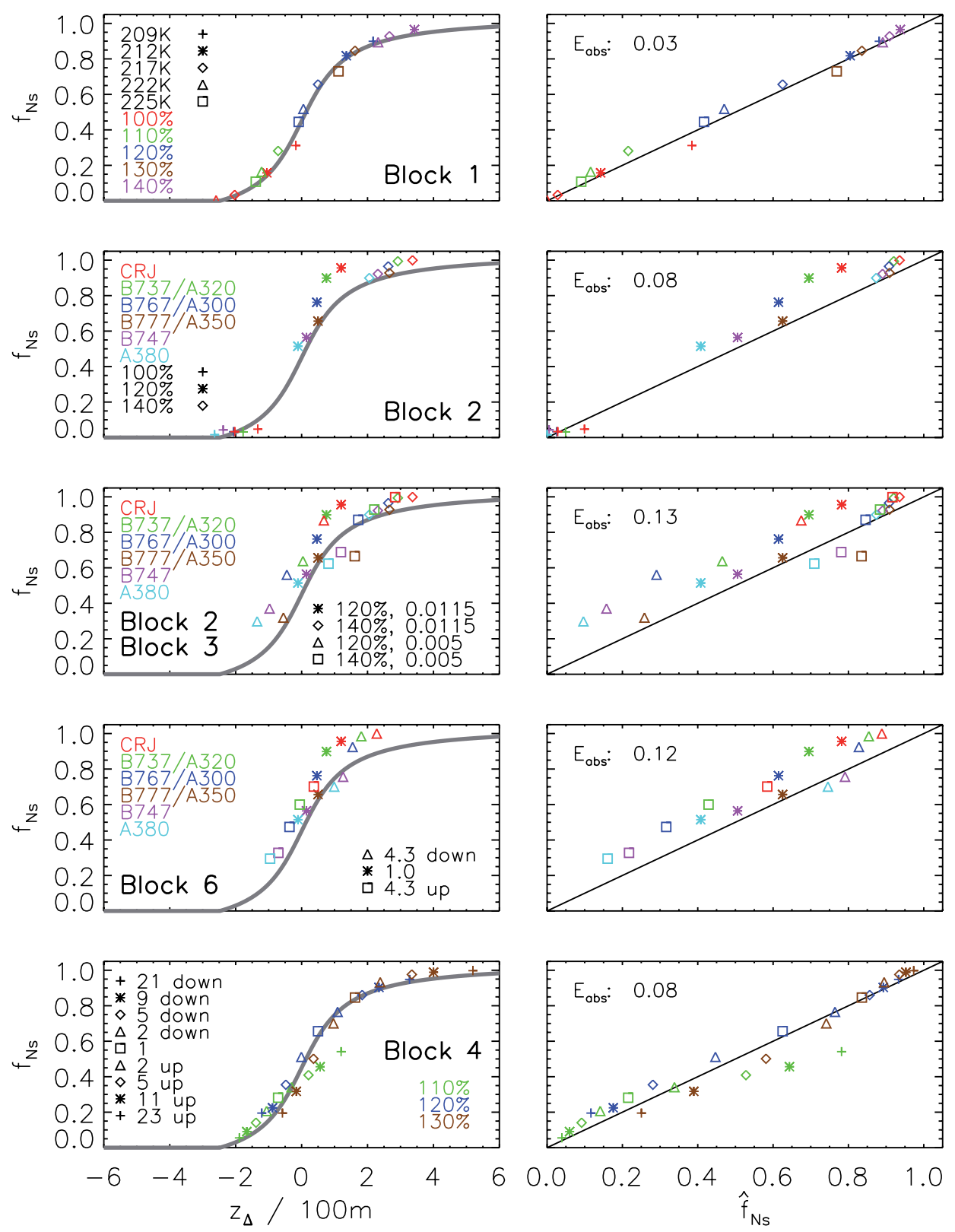

Figure 5. Left: relationship between simulated survival fraction $f_{\mathrm{Ns}}$ and $z_{\Delta}$. The grey curve shows the fit function $\hat{a}$ as defined in Eq. (9). Right: relationship between simulated survival fraction $f_{\mathrm{Ns}}$ and approximated survival fraction $\hat{f}_{\mathrm{Ns}}$. The black line shows the 1-1 line. Each row shows a subset of simulations taken from various simulation blocks defined in Table A2. For example, the first row shows simulations of block 1, where $\mathrm{RH}_{\mathrm{i}}$ and $T_{\mathrm{CA}}$ are varied. The legend in the plot provides a list of the symbols and colours, which uniquely define the simulations parameters of each plotted data point. The root mean square of the absolute error $\hat{f}_{\mathrm{Ns}}-f_{\mathrm{Ns}}$ is denoted as $E_{\mathrm{abs}}$ and given for each subset.

Finally, we provide the values of the fit parameters that were used for the computation of $z_{\Delta}$ and $\hat{a}$ :

$\beta_{0}=0.4$,

$\beta_{1}=1.19$,

$\alpha_{0}=-1.35$,

$$
\begin{aligned}
& \alpha_{\mathrm{desc}}=0.6, \\
& \gamma_{\mathrm{atm}}=0.18, \\
& \gamma_{\mathrm{emit}}=0.18 .
\end{aligned}
$$

$\alpha_{\text {atm }}=1.7 \times \mathrm{EI}_{\text {iceno }}^{*}-\gamma_{\text {atm }}$,

$\alpha_{\text {emit }}=1.15 \times \mathrm{EI}_{\text {iceno }}^{*}-\gamma_{\mathrm{emit}}$, 
We determined these values by minimising the bias and standard deviation of the absolute and relative errors. Note that we did not apply a formal optimisation algorithm to find an optimal solution. We rather made a subjective trade-off between minimising the four error parameters.

Splitting the analysis into two separate length scales $z_{\text {atm }}$ and $z_{\text {emit }}$, implicitly assumed linearity in $e_{\mathrm{s}}$, which is not the case. Using the combined length scale $z$ buffer defined by

$\left(1+s_{\mathrm{i}}\right) \frac{e_{\mathrm{S}}\left(T_{\mathrm{CA}}\right)}{R_{\mathrm{v}} T_{\mathrm{CA}}}+\rho_{\mathrm{emit}}=\frac{e_{\mathrm{s}}\left(T_{\mathrm{CA}}+\Gamma_{\mathrm{d}} z_{\text {buffer }}\right)}{R_{\mathrm{v}}\left(T_{\mathrm{CA}}+\Gamma_{\mathrm{d}} z_{\text {buffer }}\right)}$

may be more physically plausible and, indeed, $z_{\text {buffer }}$ is up to $15 \%$ smaller than the sum of $z_{\text {atm }}$ and $z_{\text {emit }}$. However, the main purpose is to design a parametrisation that approximates the simulated values the best. We found that the ansatz with two separate length scales allowed us to design a better parametrisation, as the weights $\alpha_{\text {atm }}$ and $\alpha_{\text {emit }}$ are individually adjustable.

The definitions of $z_{\mathrm{atm}}$ and $z_{\text {emit }}$ (or $z_{\text {buffer }}$ ) rely on equating water vapour concentrations. One may argue that mixing ratios (and not concentrations) are conserved during adiabatic processes. From this follows the conservation of $e_{\mathrm{S}}(T) / T^{\kappa}$ with $\kappa=3.5$ instead of $e_{\mathrm{S}}(T) / T$.

As most of the variability in $z_{\mathrm{atm}}$ and $z_{\mathrm{emit}}$ comes from $e_{\mathrm{S}}(T)$, using modified length scale definitions (with $\kappa$ ) would not improve the parametrisation.

\subsection{Contrail depth}

The determination of the contrail depth was exemplified in Sect. 2. Table A2 lists $H$ values for all simulations. Clearly, $H$ depends on $z_{\text {desc }}$. The farther the vortices descend, the deeper the contrails can be (Fig. 2 right). On the other hand, the contrails may not reach the full vertical extent. In particular for slight supersaturations, the primary wake runs dry and all ice crystals in it are lost, as shown in Fig. 2 left. Thus, the parametrisation for $H$ takes into account the combined effects of wake vortex descent and crystal loss.

Figure 6 left shows the relationship between $H / \hat{z}_{\text {desc }}$ and $\widetilde{f}_{\mathrm{Ns}}$. Note that $H$ is the contrail depth determined from the model simulations, whereas $\hat{z}_{\text {desc }}$ and $\widetilde{f}_{\mathrm{Ns}}$ are parametrisations ( $\widetilde{f}_{\mathrm{Ns}}$ is similar to $\hat{f}_{\mathrm{Ns}}$ and will be defined later). Using parametrised rather than simulated values for $z_{\text {desc }}$ and $f_{\mathrm{Ns}}$ allows us to derive a parametrisation for $H$ that is based only on available data. We suggest a piecewise linear function $\hat{b}(x)$ as an approximation for $H / \hat{z}_{\text {desc }}$ as indicated by the grey curve.

Then the parametrised contrail height $\hat{H}$ is given by

$\hat{H}=\hat{z}_{\operatorname{desc}} \times \hat{b}\left(\widetilde{f}_{\mathrm{Ns}}\right)$,

where

$\hat{b}(x)=\left\{\begin{array}{cc}\eta_{1} x & x \in\left[0, x_{\mathrm{s}}\right] \\ \eta_{2} x+\left(\eta_{1}-\eta_{2}\right) x_{\mathrm{S}} & x \in\left[x_{\mathrm{s}}, 1\right]\end{array}\right.$

with $\eta_{1}=6, \eta_{2}=0.15$ and $x_{\mathrm{s}}=0.2$.
The piecewise definition reflects the fact that contrail depth changes only slightly with large survival fractions and is much stronger once a major fraction of the ice crystals is lost. Thus, the definition is split into two parts (above and below $x_{\mathrm{s}}$ ) and the slope of the approximation is much higher in the $x<x_{\mathrm{s}}$ part; i.e. $\eta_{1}$ is much larger than $\eta_{2}$.

Note that $\hat{b}$ can achieve values greater than unity implying that $\hat{H}$ can be greater than $\hat{z}_{\text {desc }}$. This is reasonable as the contrail extends above the formation altitude on the one hand. Moreover, $z_{\text {desc }}$ was determined by finding the centre of the primary wake whereas the contrail bottom is defined by the lower end of the plume.

Finally, we define $\widetilde{f}_{\mathrm{Ns}}$, which is used in Eq. (12) and is in one aspect different from $\hat{f}_{\mathrm{Ns}}$. Section 2 discussed that a variation of $\mathrm{EI}_{\text {iceno }}$ has a small impact on the contrail mass and depth. The impact on crystal loss is however large and the values of $\hat{f}_{\mathrm{Ns}}$ are distributed over a large range, as can be seen in bottom right panel of Fig. 5 . In the definition of $\widetilde{f}_{\mathrm{Ns}}$ we exclude the $\mathrm{EI}_{\text {iceno }}$ effect by simple means. We take the original definition for $\hat{f}_{\mathrm{Ns}}$ and simply set $\gamma_{\mathrm{atm}}=\gamma_{\mathrm{emit}}=0$.

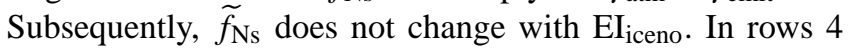
and 5 of Fig. 6 the various symbols of a specific colour all lie on one vertical line. From Eq. (12) follows that $\hat{H}$ does not depend on $\mathrm{EI}_{\text {iceno. }}$.

Analogous to Fig. 5, the right panel of Fig. 6 shows a scatter plot, now contrasting $H$ vs. $\hat{H}$. Again, root mean squares of the absolute errors are supplied in the figure. They are around $50 \mathrm{~m}$ or below for each subset, which again proves the suitability of the proposed parametrisation.

\subsection{Contrail width}

Contrail width is a parameter whose choice is not as critical for the later evolution as that of contrail depth and ice crystal number. The horizontal spreading rate of a contrail is roughly given by $\dot{W}=H \times s$. For typical values $H=400 \mathrm{~m}$ and vertical wind shear $s=0.005 \mathrm{~s}^{-1}$ (considering only the component normal to contrail length axis), the width increases by $1 \mathrm{~km}$ every $8 \mathrm{~min}$. Thus, an uncertainty in the initial width translates into a small offset in the assumed contrail age, which becomes unimportant when looking at contrailto-cirrus evolution over several hours.

The determination of the contrail width is based on the evaluation of transverse profiles of ice crystal mass after the vortex phase, which are depicted in Fig. 7. The distributions resemble roughly Gaussian distributions (at least in the absence of a sheared cross-wind and when averaged along the flight direction).

For a B777 aircraft (left panel), most of the ice mass is confined to a $150 \mathrm{~m}$ broad band centred around the aircraft body. Towards the end of their lifetime, the vortex tubes meander and some ice is laterally even farther dislocated. This effect is not apparent, when all ice of the primary wake has been lost before this stage $\left(\mathrm{RH}_{\mathrm{i}} \leq 110 \%\right)$. The middle panel shows a modest dependence of contrail width on aircraft 

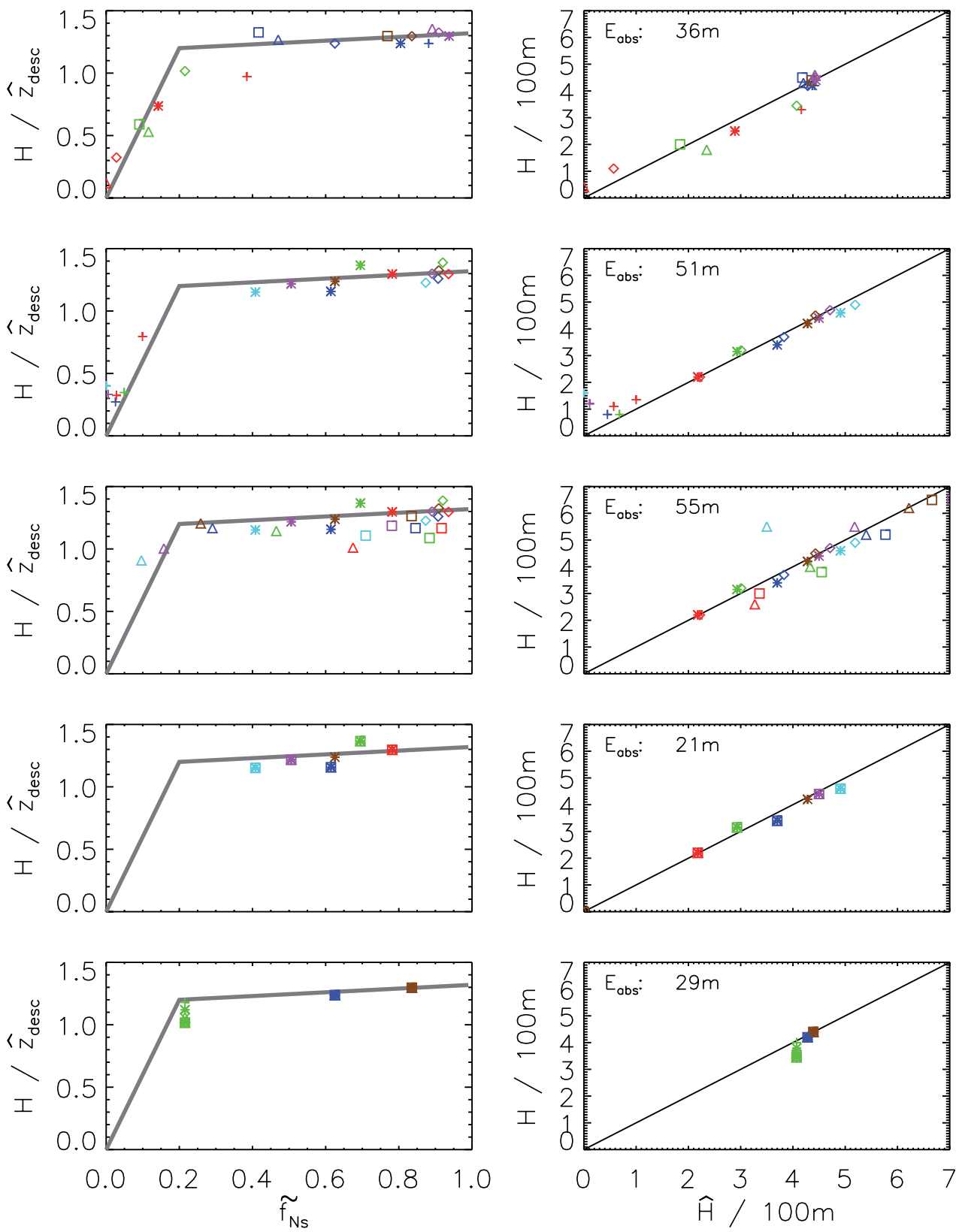

Figure 6. Left: relationship between simulated contrail depth $H$ over approximated $\hat{z}_{\text {desc }}$ and approximated survival fraction $\tilde{f}_{\text {Ns }}$. The grey curve shows the fit function $\hat{b}$ as defined in Eq. (13). Right: relationship between simulated contrail depth $H$ and approximated contrail depth $\hat{H}$. The root mean square of the absolute error $\hat{H}-H$ is denoted as $E_{\text {abs }}$ and given for each subset. The simulation subsets and the layout are analogous to Fig. 5.

type. The smaller the aircraft wing span is, the smaller is the distance between the two vortex centres and exhaust plumes and the narrower is the final contrail. In the case with weaker stratification (right panel), the formation of vortex rings is pronounced, the vortex tubes oscillate more strongly and the contrail is broader.

Thus, 3-D dynamical phenomena like the well-known Crow instability of the trailing vortices (Crow, 1970) lead to substantial variations along the flight direction, even if the environmental conditions are homogeneous in this direction, as exemplified by Lewellen et al. (1998) and Unterstrasser et al. (2014) for conserved exhaust species, and by Lewellen and Lewellen (2001) and U2014 for contrails.

This also implies that the contrail width varies along flight direction and attains its maximum values only in certain segments along the flight direction. Figure 8 depicts cross sections of number concentrations from two slices, which are only $60 \mathrm{~m}$ apart. Slice B contains a factor of 2.5 more ice 


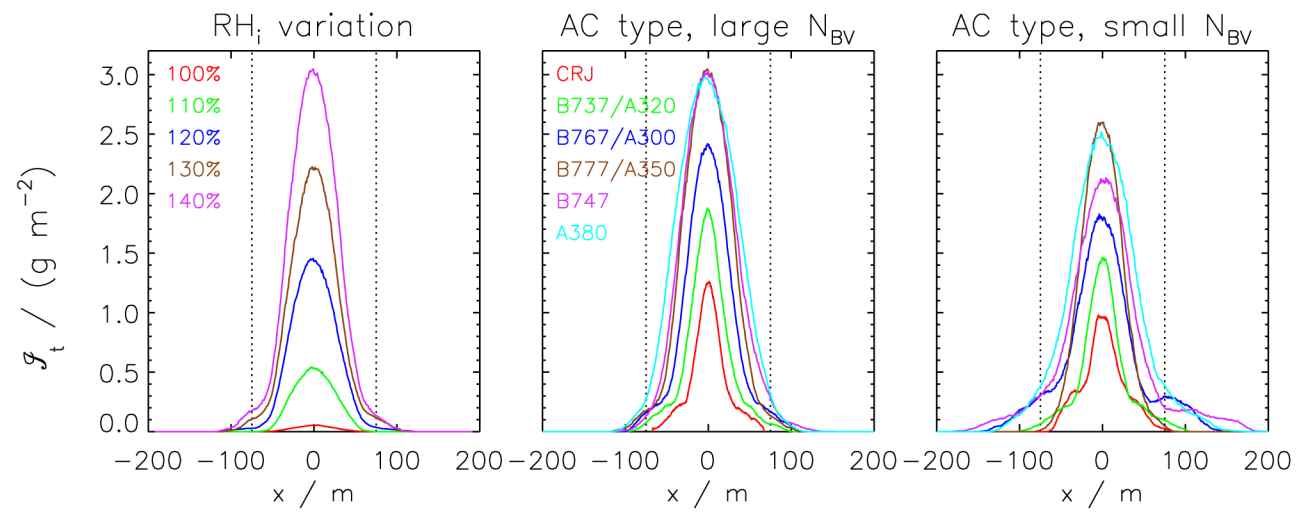

Figure 7. Transverse profiles of ice crystal mass for various simulation subsets. The profiles show ice water content integrated over the vertical direction and averaged along flight direction after $4 \mathrm{~min}$. The left panel shows a $\mathrm{RH}_{\mathrm{i}}$ variation for a B777-type aircraft; the middle/right panel shows a variation of aircraft type for strong/weak stratification $\left(N_{\mathrm{BV}}=1.15 \times 10^{-2}\right.$ and $\left.0.5 \times 10^{-2} \mathrm{~s}^{-1}\right)$ at $\mathrm{RH}_{\mathrm{i}}=120 \%$. The simulations are listed in Block 1, 2 and 3 of Table A2, respectively. The dotted vertical lines indicate the $W=150 \mathrm{~m}$ approximation.

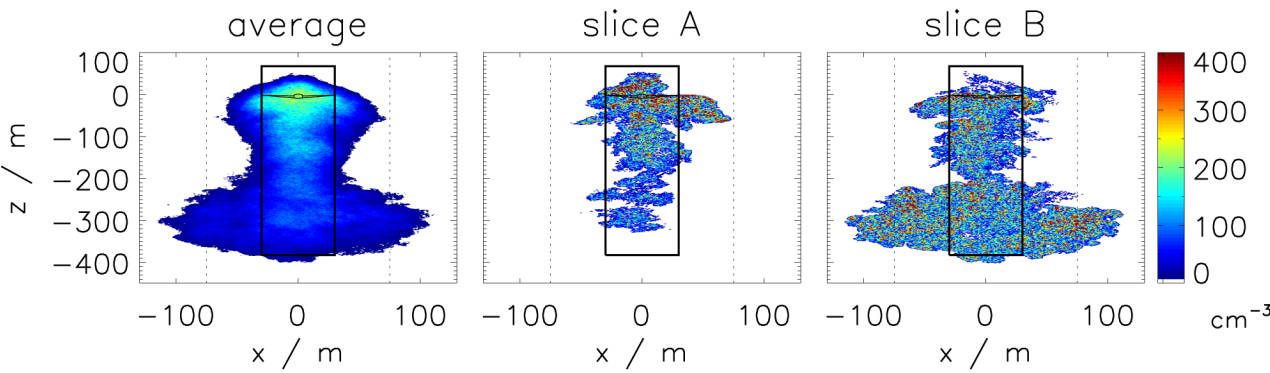

Figure 8. Ice crystal number concentrations in a plane normal to the flight direction after 4 min. The displayed simulation is no. 9 from Table A2. In the left panel the number concentrations are summed up along flight direction and divided by the length of the flight segment. In the middle and right panel, two slices along flight direction are extracted. The dotted vertical lines indicate the $W=150 \mathrm{~m}$-approximation (i.e. $x= \pm 75 \mathrm{~m}$ ). The black box indicates effective volume of the contrail. The box height equals the $H$ value given in Table A2. The width $W_{\text {rect }}$ is given by $A / H$, where $A$ is the longitudinally averaged cross section.

crystals than slice A. Moreover, the contrail is much broader, in particular in the lower part.

Global-scale models, in which the current parametrisation might be employed in the future, cannot resolve such subtleties as contrail intrinsic heterogeneities. Thus, we propose the simple estimate $\hat{W}=150 \mathrm{~m}$ independent of the parameter settings. A more sophisticated approximation may include dependencies on wing span and Brunt-Väisälä frequency.

In global-scale models, where the mean age of the initialised contrails is typically half the time step (which is at present times larger than the contrail age used here) one may correct for this offset in time.

If one is interested in deriving number concentrations from the parametrisations of $N, H$ and $W$, a more suitable width parametrisation is presented in Sect. 4.3.

\section{Applications}

\subsection{Test case of a soot reduction experiment}

The number of generated ice crystals $N_{\text {form }}$ depends on the number of emitted soot particles $N_{\text {soot }}$. The higher the corresponding emission indices are, the more crystals are lost during the vortex phase. We apply our derived parametrisation to determine an average survival fraction for $\mathrm{EI}_{\text {iceno }}=$ $10^{15}, 10^{14}$ and $10^{13} \mathrm{~kg}^{-1}$, respectively. The average is taken over a 4-D cube varying relative humidity (100-140\%), temperature (210-226 K), Brunt-Väisälä frequency (0.006$\left.0.014 \mathrm{~s}^{-1}\right)$ and wing span (20-84 m). Using the empirical relationships given by Eqs. (A5), (A9) and (A10), the wing span determines all relevant aircraft properties. For each parameter, the survival fraction is evaluated for nine values equally distributed over the given range (totalling $9^{4}$ combinations). About 29,55 and $75 \%$ of the ice crystals survive for $\mathrm{EI}_{\text {iceno }}=10^{15}, 10^{14}$ and $10^{13} \mathrm{~kg}^{-1}$. Thus, a $\mathrm{EI}_{\text {iceno }}$ reduction from $10^{15}$ down to $10^{14} \mathrm{~kg}^{-1}$ (or $10^{14}$ down to $10^{13} \mathrm{~kg}^{-1}$ ) implies only a factor of 5.3 (or 7.4) reduction of the ice crys- 


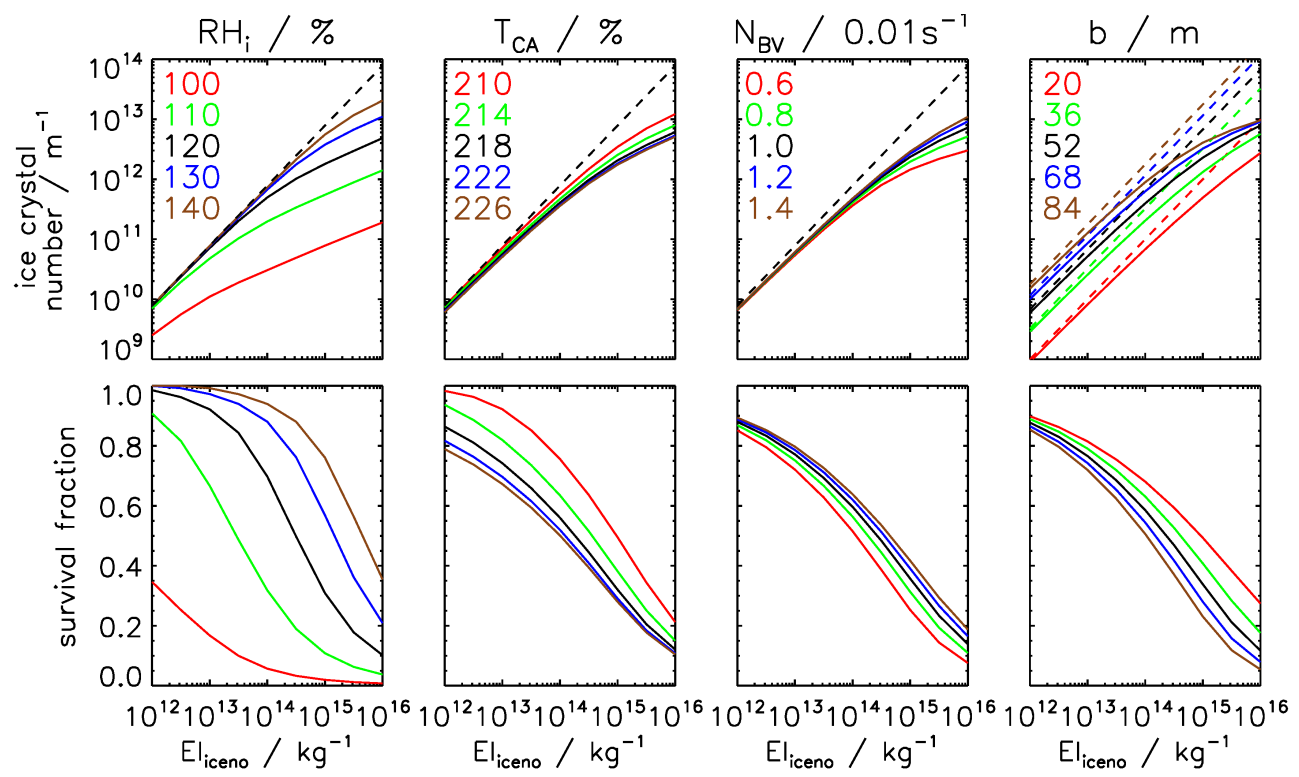

Figure 9. Sensitivity of ice crystal loss to $\mathrm{EI}_{\mathrm{iceno}}$ for various values of $\mathrm{RH}_{\mathrm{i}}, T, N_{\mathrm{BV}}$ and $b$ (from left to right). See legend for the colour coding. Top row: ice crystal number per metre of flight path before and after the vortex phase (dashed and solid curves). Note that the initial ice crystal number depends only on $b$ and $\mathrm{EI}_{\text {iceno }}$ (following Eq. A10). Hence, only one dashed curve is shown in the columns for $\mathrm{RH}_{\mathrm{i}}, T$ and $N_{\mathrm{BV}}$, respectively. Bottom row: survival fraction.

tal number in the end. An initial factor 100 reduction gives 40 times fewer ice crystals in the end. So far, all $9^{4}$ data points (variation over $\mathrm{RH}_{\mathrm{i}}, T_{\mathrm{CA}}, N_{\mathrm{BV}}$ and $b$ ) are equally weighted, which could certainly be refined in the future.

Now more detailed sensitivity tests follow, where we analyse the behaviour of an $\mathrm{EI}_{\text {iceno }}$ variation for certain subsets of the parameter space. For this, we take the average over three out of the four dimensions of the 4-D cube and show the $\mathrm{EI}_{\text {iceno }}$ dependence for different values of the fourth parameter. Figure 9 shows the $\mathrm{EI}_{\mathrm{iceno}}$ dependence of the crystal loss for different values of $\mathrm{RH}_{\mathrm{i}}, T_{\mathrm{CA}}, N_{\mathrm{BV}}$ and $b$ (from left to right). $\mathrm{EI}_{\text {iceno }}$ runs from $10^{12}$ to $10^{16} \mathrm{~kg}^{-1}$. The solid lines in the top row depict the number of surviving ice crystals. The average over a 3 -D cube $\bar{N}_{\text {surv }}=\sum_{i, j, k=0}^{8} \hat{N}_{\text {surv }}(i, j, k) / 9^{3}$ is shown, where $\hat{N}_{\text {surv }}=N_{\text {form }} \times \hat{f}_{\mathrm{Ns}}$ and $i, j$ and $k$ are generic indices for three selected parameters. Similarly, the dashed lines show the initial ice crystal number $\bar{N}_{\text {form }}=$ $\sum_{i, j, k=0}^{8} N_{\text {form }}(i, j, k) / 9^{3}$. The bottom row shows the survival fraction $\bar{f}_{\mathrm{Ns}}=\sum_{i, j, k=0}^{8} \hat{f}_{\mathrm{Ns}}(i, j, k) / 9^{3}$. As expected, $\bar{N}_{\text {surv }}$ and $\bar{f}_{\mathrm{Ns}}$ increase with increasing $\mathrm{RH}_{\mathrm{i}}$, decreasing $T_{\mathrm{CA}}$ and increasing $N_{\mathrm{BV}}$. The dependence on wing span is more complicated. Even though $\bar{f}_{\mathrm{Ns}}$ decreases with increasing $b$, $\bar{N}_{\text {surv }}$ does increase, as this is overcompensated by an increase in $N_{\text {form }}$ (following Eq. A10). Now we turn the attention to the $\mathrm{EI}_{\text {iceno }}$ dependence. Clearly, $\bar{f}_{\mathrm{Ns}}$ decreases with increasing $\mathrm{EI}_{\mathrm{iceno}}$. The dependence on $\mathrm{EI}_{\text {iceno }}$ is qualitatively similar for all values of $T_{\mathrm{CA}}, N_{\mathrm{BV}}$ and $b$. However, for different $\mathrm{RH}_{\mathrm{i}}$ values, the function $\bar{f}_{\mathrm{Ns}}\left(\mathrm{EI}_{\text {iceno }}\right)$ looks qualitatively different. We can divide the analysed $\mathrm{EI}_{\text {iceno }}$ range in a low
$\mathrm{EI}_{\text {iceno }}$ regime and a high $\mathrm{EI}_{\text {iceno }}$ regime. In the low $\mathrm{EI}_{\text {iceno }}$ regime, we find a strong dependence of $\bar{f}_{\mathrm{Ns}}$ on $\mathrm{EI}_{\text {iceno }}$ for low supersaturation and a weak dependence for high supersaturation. In the high $\mathrm{EI}_{\text {iceno-regime, it is the other way round. The }}$ slope of $\bar{f}_{\mathrm{Ns}}\left(\mathrm{EI}_{\mathrm{iceno}}\right)$ is steeper, the higher the supersaturation is.

Biofuels cause lower soot emissions with a likely impact on $N_{\text {form }}$. As the initial differences are reduced during the vortex phase, mitigation studies neglecting those effects may overestimate the effect of biofuels.

\subsection{Sensitivity to input parameters}

In this section, we analyse the sensitivity of $N$ and $H$ to the various input parameters of the parametrisation. We evaluate

$\hat{N}_{\text {surv }}=N_{\text {form }} \times \hat{f}_{\mathrm{Ns}}$

and $\hat{H}$ for the 4-D cube as defined above and take the average over three out of the four dimensions. Figure 10 shows the ice crystal number and the contrail depth after the vortex phase as a function of the various input parameters. We separate between scenarios with $\mathrm{EI}_{\text {iceno }}=10^{15}$ and $10^{14} \mathrm{~kg}^{-1}$. The variation in $N$ is due to variations in $N_{\text {form }}$ and $\hat{f}_{\mathrm{Ns}}$. Note that $N_{\text {form }}$ depends only on wing span $b$ and $\mathrm{EI}_{\text {iceno }}$ following Eq. (A10), whereas $\hat{f}_{\mathrm{Ns}}$ depends on all five parameters. In combination, we find that $N$ depends most strongly on $\mathrm{EI}_{\text {iceno }}$ and $\mathrm{RH}_{\mathrm{i}} . b, T_{\mathrm{CA}}$ and $N_{\mathrm{BV}}$ (in this order) have a smaller impact.

The contrail depth depends most strongly on $\mathrm{RH}_{\mathrm{i}}$ and $b$ and to a lesser extent on $T_{\mathrm{CA}}$ and $N_{\mathrm{BV}}$. According to the 
design of our parametrisation, the contrail depth does not depend on $\mathrm{EI}_{\text {iceno. }}$.

The sensitivity analyses reveal the most significant parameters for the determination of ice crystal number and contrail depth. It gives an estimate on how uncertainty in the input parameters translates into uncertainties in the output parameters.

Concerning the crystal loss determination, $\mathrm{EI}_{\mathrm{iceno}}$ and $\mathrm{RH}_{\mathrm{i}}$ should be well characterised in some future application of the parametrisation, whereas for $N_{\mathrm{BV}}$ and $T_{\mathrm{CA}}$ it may be sufficient to have a rough estimate or to assume some default values.

\subsection{Implications on number concentrations}

From the parametrisations of $N, H$ and $W$ one may derive the mean ice crystal number concentration $\hat{n}_{\text {mean }}=$ $\hat{N}_{\text {surv }} /(\hat{H} \hat{W})$ of a specific contrail. Hereby, we assume that the ice crystals are spread over a rectangular cross section with width $\hat{W}$ and height $\hat{H}$. Figure 11 contrasts $\hat{n}_{\text {mean }}$ with the simulated values of $n_{\text {mean }} . n_{\text {mean }}$ is given by $N_{\text {surv }} / A$, where $A$ is the actual cross sectional area of a contrail (averaged along flight direction). We find that $\hat{n}_{\text {mean }}$ underestimates the simulated values by roughly a factor of 5 (left panel). The main reason for this bias is that the parametrised area $\hat{A}=\hat{W} \hat{H}$ overestimates the real area $A$ by around the same factor. In Fig. 8 , the black boxes show the area-equivalent rectangle for this specific contrail with width $W_{\text {rect }}=A / H . W_{\text {rect }}$ is much smaller than the actual width of the contrail. The parametrised number concentrations will become more realistic, if we prescribe an areaequivalent width $W_{\text {rect }}=0.63 b$ and use $\hat{A}=W_{\text {rect }} \hat{H}$ instead. The constant 0.63 is chosen such that the expectation value of $\Delta n_{\text {mean }}=\hat{n}_{\text {mean }}-n_{\text {mean }}$ is zero. The right panel 11 shows a much better agreement of $\hat{n}_{\text {mean }}$ with $n_{\text {mean }}$. The standard deviation of the relative error $2 \Delta n_{\text {mean }} /\left(\hat{n}_{\text {mean }}+n_{\text {mean }}\right)$ is around $50 \%$. The prediction of $n_{\text {mean }}$ is more uncertain than that of $N$ or $H$, as the uncertainties add together. The parametrised number concentrations are similarly realistic when we use $W_{\text {rect }}=36 \mathrm{~m}$ (not shown; again the expectation value is zero and the standard deviation is $55 \%$ ).

Depending on the purpose the parametrisation is applied for, we recommend using either the $\hat{W}$ definition from Sect. 3.4 or the $W_{\text {rect }}$ definition from this section.

As the contrails get diluted, mean concentrations decrease over time. Evaluating the simulated $n_{\text {mean }}$ at $t=3 \mathrm{~min}$ instead of at $t=5 \mathrm{~min}$, we obtain 1.65 times higher values (with a standard deviation of 0.23 ).

Analogous to the analyses in Sect. 4.2, Fig. 10 (middle) shows the sensitivity of $\hat{n}_{\text {mean }}$ (after $5 \mathrm{~min}$ ) to the various input parameters of the parametrisation. We find a dominant impact of $\mathrm{EI}_{\text {iceno }}$ and $\mathrm{RH}_{\mathrm{i}} . b, T_{\mathrm{CA}}$ and $N_{\mathrm{BV}}$ appear to be less important. Using $W_{\text {rect }}=36 \mathrm{~m}$ instead of $W_{\text {rect }}=0.63 \mathrm{~b}$, various opposing trends do not cancel out and the sensitivity to $b$ is larger (not shown).
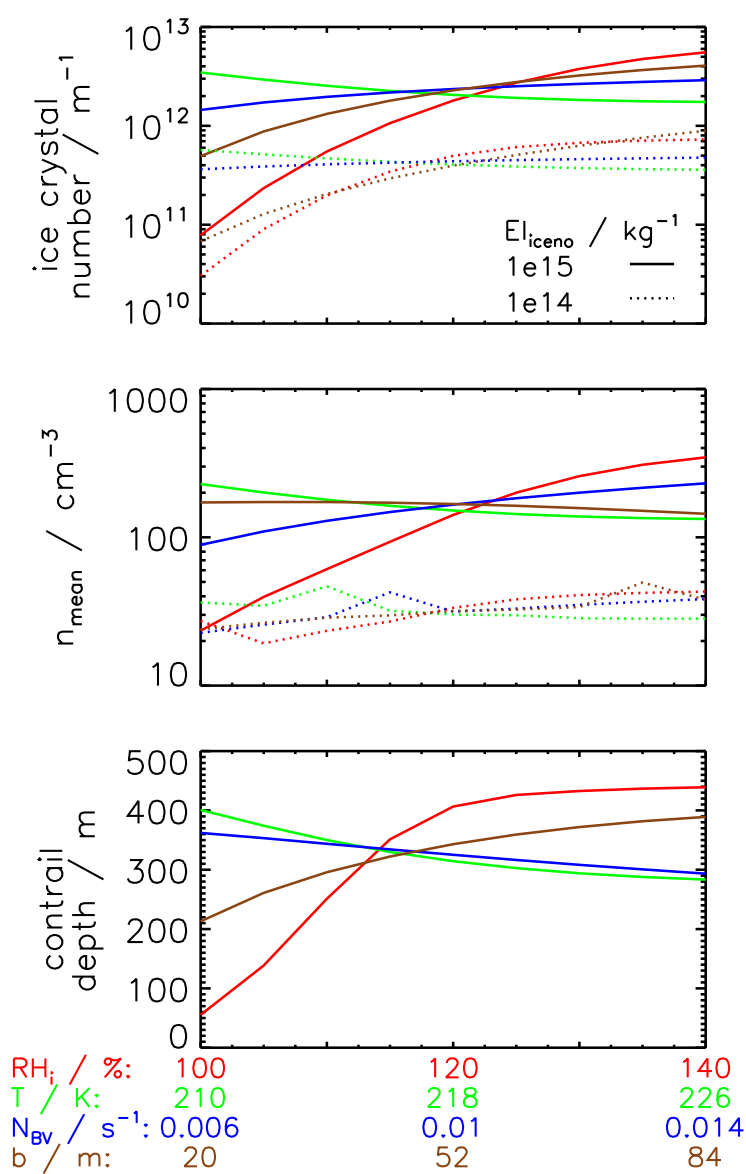

Figure 10. Ice crystal number per metre of flight path (top), mean ice crystal number concentration (middle) and contrail depth (bottom) after the vortex phase as a function of $\mathrm{RH}_{\mathrm{i}}, T, N_{\mathrm{BV}}$ or $b$. $\mathrm{EI}_{\text {iceno }}$ is $10^{15}$ or $10^{14} \mathrm{~kg}^{-1}$. The contrail depth parametrisation does not depend on $\mathrm{EI}_{\text {iceno. }}$.

Deriving analogous relations for ice water content or optical depth would be desirable. As we do not parametrise the contrail ice mass, this cannot be achieved with the present parametrisation.

\section{Discussion}

In the preceding section we introduced parametrisations of crystal loss and contrail depth, which take into account the effect of the most important parameters, namely temperature at cruise altitude $T_{\mathrm{CA}}$, ambient relative humidity $\mathrm{RH}_{\mathrm{i}}$, BruntVäisälä frequency $N_{\mathrm{BV}}$, aircraft properties (defining the initial wake vortex properties and the water vapour emission) and ice crystal emission index $\mathrm{EI}_{\text {iceno. }}$.

\subsection{Further sensitivities}

Several further parameters may affect the early contrail evolution. Their importance, which has been partly investigated 

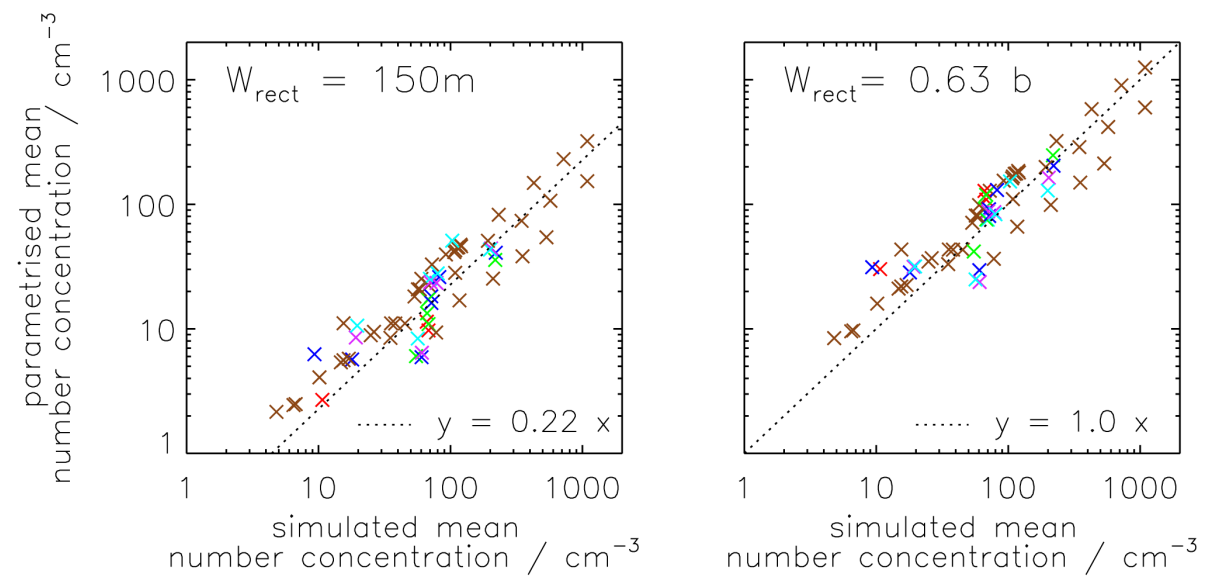

Figure 11. Simulated vs. parametrised mean ice crystal number concentration. In the simulation, the mean is taken over all grid boxes with non-zero ice crystal number concentration. The parametrised $n_{\text {mean }}$ is given by $\hat{N}_{\text {surv }} /\left(\hat{H} W_{\text {rect }}\right)$. In the left panel, $W_{\text {rect }}$ is $\hat{W}=150 \mathrm{~m}$. In the right panel, $\hat{W}$ is $0.63 b$, where $b$ is the wing span.

by recent 3-D simulation studies, will be discussed in the following.

Stronger vertical wind shear and higher ambient turbulence potentially speed up the vortex decay. However, for stratification values typical of the upper troposphere, variations of vertical wind shear $s$ (assuming a linear wind profile) and turbulence are second-order effects and wake vortex descent, contrail height and crystal loss are fairly unaffected by such variations (Huebsch and Lewellen, 2006; Hennemann and Holzäpfel, 2011; Naiman et al., 2011; Unterstrasser et al., 2014; Picot et al., 2015). For strongly curved wind profiles (i.e. non-zero $\dot{s}$ ) vortex decay may be accelerated and the situation becomes more intricate.

Cruise altitude mainly determines the ambient temperature, pressure and air density (linked by the ideal gas law). Note that the definition of $z_{\mathrm{atm}}$ and $z_{\mathrm{emit}}$ is based on equating water vapour concentrations, which do not depend on ambient pressure/density (unlike to mixing ratios). Thus, the contrail ice mass balance and consequently crystal loss and contrail depth are in theory not affected by ambient pressure. Ambient pressure affects, for example, the diffusivity of water vapour and thus ice crystal growth (Ghosh et al., 2007; Pruppacher and Klett, 1997). But the sensitivity is too weak to be important in this case as confirmed by 2-D contrail simulations (Unterstraßer, 2008). Note that the variation of other microphysical constants (not dependent on pressure) like the deposition coefficient induce larger uncertainties (Lewellen et al., 2014).

The initial circulation $\Gamma_{0}$ of the wake vortices is inversely proportional to the air density (see Eq. A1) and changes with flight altitude. Compared to the variation of aircraft mass and wing span, the rather small changes in $\rho_{\text {air }}$ have a secondorder effect on $\Gamma_{0}$ and the wake vortex displacement $z_{\text {desc }}$. Thus, the dependence of contrail evolution on flight altitude is mainly a temperature effect.
In the simulations presented in Sect. 3 ambient relative humidity was assumed to be uniform over the entire domain. Hence, we do not cover scenarios with a thin supersaturated layer where the contrail leaves the supersaturated layer at some point during the wake vortex descent. A thin moist layer may limit the contrail depth and inhibit contrail growth in its early stage. However, such scenarios have not yet been analysed in any LES study of young contrails. So far, the impact of the depth of the supersaturated layer was only investigated for contrail-cirrus (Unterstrasser and Gierens, 2010b; Lewellen, 2014); i.e. the supersaturated layer was deep enough to contain the young contrail, yet limited the later formation of the fall streak.

All simulations discussed in Sect. 3 use the standard configuration, as we call it. Further uncertainties arise from the choice of this configuration and may have an impact on the simulated crystal loss and contrail depth. Clearly, these parameters are not reflected in our parametrisations. This includes variations of the spatial initialisation and the initial width of the ice crystal size distribution as well as the disregard of the Kelvin effect in the ice growth equation. Their effects have been discussed in detail in U2014 and the effect on contrail depth was found to be negligible and is not further discussed here. Crystal loss, however, is affected and in the following we discuss whether this downgrades the universality of our parametrisations.

Figure 12 depicts $f_{\mathrm{Ns}}$ of these sensitivity simulations (coloured symbols) and contrasts them with the simulations with the standard configuration (grey symbols, all those simulations that were originally depicted in Fig. 5).

In the default configuration, UG2014 and U2014 use a simple spatial initialisation; i.e. the crystal number concentrations are constant inside the two initial plumes. Following the approach of Lewellen et al. (2014) and Naiman et al. (2011), Gaussian-like distributions of ice crystal num- 

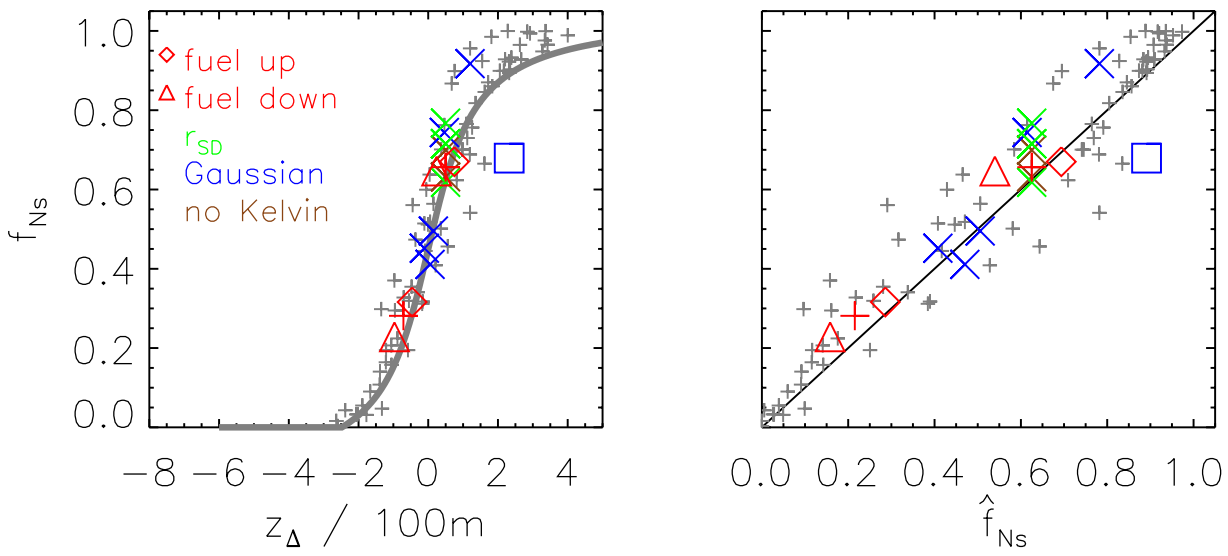

Figure 12. Plots as in Fig. 5. Grey symbols show all simulation data from Fig. 5. The coloured symbols show sensitivity simulations (see legend) not yet discussed.

ber concentrations were alternatively prescribed (see blue symbols). The effect on the crystal loss extent was often low (originally discussed in Fig. 7 in UG2014). In one particular case with high temperature and relative humidity, the crystal loss was stronger, once a Gaussian distribution was used (originally discussed in Fig. 13 of U2014; blue square in our Fig. 12). If a Gaussian distribution is more realistic, the survival rates may be overestimated for high supersaturations.

Based on the assumption of spherical ice crystals, the standard configuration includes a correction of the local relative humidity over a curved surface (Kelvin effect) in the ice crystal growth equation. It is not clear how physically plausible this is, as ice crystals are never perfect spheres. When we switch off the Kelvin correction, fewer ice crystals are lost (brown symbols), as expected.

In U2014, the initial width of the ice crystal size distribution was varied, as it cannot be measured or numerically determined accurately enough (see discussion in Unterstrasser and Sölch, 2010). The narrower the SD chosen is, the fewer ice crystals lost (green symbols). Note that Lewellen et al. (2014) reports a weaker sensitivity to this parameter which may be connected to the fact that the spatial initialisation of the ice crystals and the assumed initial wake age differ between their study and ours.

Ideally, the initialisation of the vortex phase simulations would use input from 3-D jet phase simulations that model the contrail formation with a detailed ice activation scheme and which could provide 3-D fields of the ice crystal size distribution, water vapour, temperature and velocity (only to name the most important ones). Unfortunately, such simulations are not available. Presently, 3-D jet phase simulations employ simplified ice activation schemes (Paoli et al., 2004, 2013; Garnier et al., 2014), whereas detailed plume microphysics are embedded in 0-D models with prescribed plume dilution (Kärcher and Yu, 2009; Yu and Turco, 1998).

Overall, we can state that the new data points (of the sensitivity simulations) lie within the spread of data points with the standard configuration. This implies that the uncertainties/biases due to the numerical configuration are smaller than the deviations of the simulation results from the fit function. This suggests that the uncertainties are acceptable given the accuracy of the parametrisation.

\subsection{Comparison with other LES models}

The early contrail evolution has been studied by several groups with different LES codes in the past (Huebsch and Lewellen, 2006; Lewellen et al., 2014; Naiman et al., 2011; Paugam et al., 2010; Picot et al., 2015). Qualitatively, the sensitivity trends are similar for most studies (except for one outlier model discussed below) and confirms the reliability of the model results. Nevertheless, quantitative comparisons between the various models were always hampered by the fact that in each study parameter variations started from different base states. This means that differences in the simulated contrail properties could always be attributed to slightly different input parameters and as a consequence possible systematic differences may be overlooked. Our approach offers an ideal framework to make a more in-depth intercomparison between the models and further check whether the results from other LES models support our proposed parametrisation. Block 7 of Table A2 lists input parameters as well as the values for $f_{\mathrm{Ns}}$ and $H$ from several simulations of the abovementioned studies. Analogous to the evaluation in Sect. 3, we compute $z_{\Delta}$ and $\hat{z}_{\text {desc }}$ for the given input parameters and derive the approximated values for the survival fraction and contrail depth. Those are then compared to the simulated values (see Fig. 13). First we discuss the survival rates depicted in the top row. All EULAG-LCM results from UG2014 and U2014 are plotted as light grey symbols; results from other groups are plotted with coloured symbols. The data set includes a combined variation of $T_{\mathrm{CA}}$ and $\mathrm{RH}_{\mathrm{i}}$ (red crosses) as well as a wide range $\mathrm{EI}_{\text {iceno }}$ variation (red plus symbols) by Lewellen et al. (2014). We find that their simulated val- 

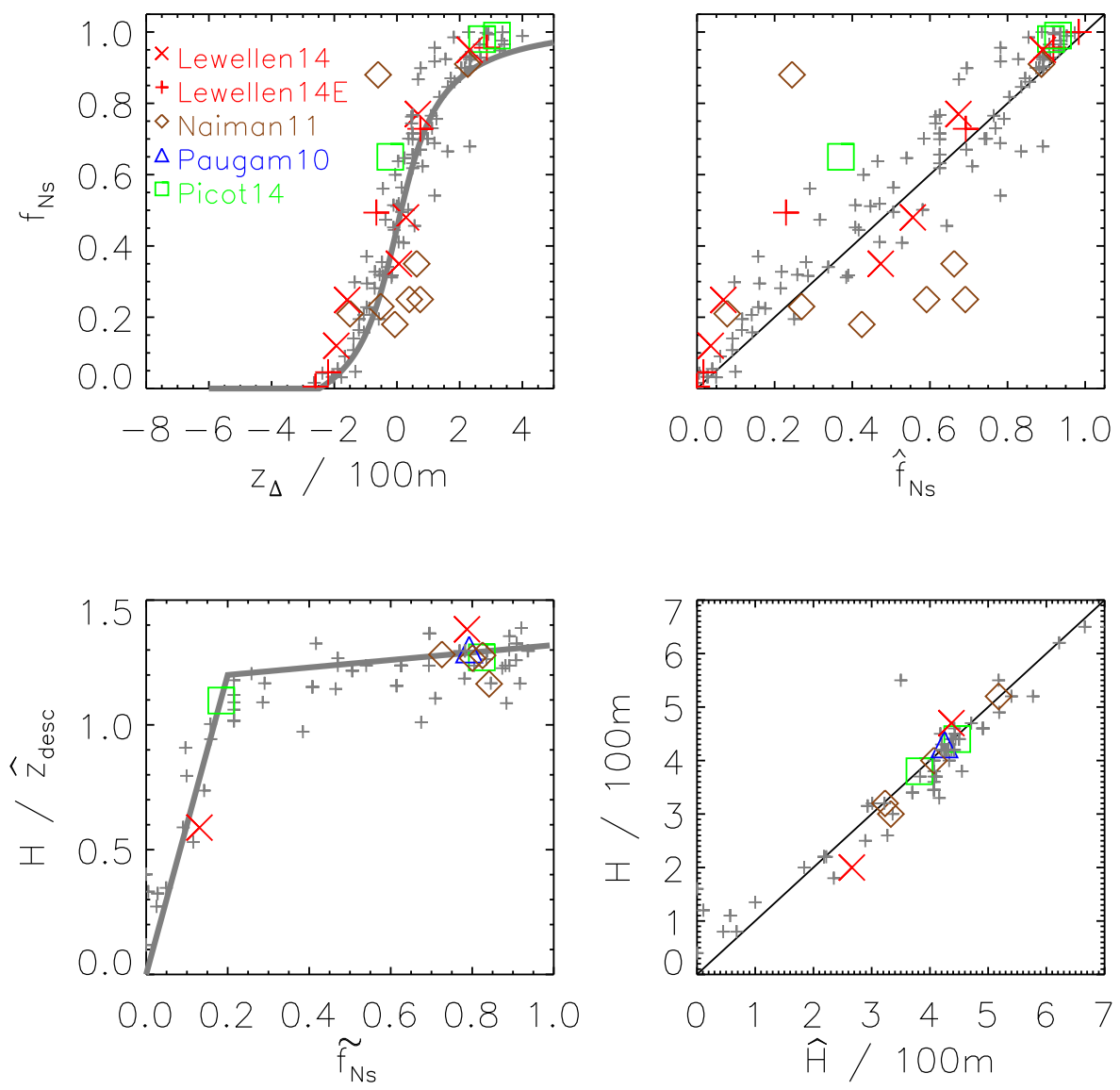

Figure 13. Top row: plot as in Fig. 5. Bottom row: plot as in Fig. 6. Grey symbols show all simulation data from Figs. 5 and 6. The coloured symbols show simulation results from other LES codes. Data are taken from Lewellen et al. (2014), Naiman et al. (2011), Paugam et al. (2010) and Picot et al. (2015).

ues for the survival fraction nicely follows our parametrisation; i.e. the red symbols are not farther away from the 1-to1 line (in the right hand side panel) than the grey symbols. Similarly, the few data points by Picot et al. (2015) agree reasonably well with our parametrisation. This demonstrates the consistency between those models and also the robustness of the $f_{\mathrm{Ns}}$ parametrisation. Now we turn our attention to the survival rates simulated by Naiman et al. (2011) (brown diamonds). Section 3.5 of U2014 already pointed out that the observed trends in several sensitivity studies of Naiman et al. (2011) disagreed with all other models and appeared implausible. The present analysis confirms this and reveals an inconsistency of their data with our $f_{\mathrm{Ns}}$-parametrisation. Obviously, their survival rates scatter strongly and there is no correlation between their $f_{\mathrm{Ns}}$ values and the predicted values $\hat{f}_{\text {Ns. }}$.

As a next step, we compare the contrail depth values of the various models (bottom row of Fig. 13). Again, the data points from the other groups are added in colour. From Picot et al. (2015) (green squares) and Huebsch and Lewellen (2006) (red crosses), $H$ values are available for $\mathrm{RH}_{\mathrm{i}}=130 \%$ and $\mathrm{RH}_{\mathrm{i}}=110 \%$ simulations. For the other studies, only results of $\mathrm{RH}_{\mathrm{i}}=130 \%$ simulations are available. For all $\mathrm{RH}_{\mathrm{i}}=130 \%$ cases, the contrails reach their full vertical extent and accordingly the ratio $H / \hat{z}_{\text {desc }}$ is around $1.2-1.4$ (left panel) as in our EULAG-LCM simulations. For the $\mathrm{RH}_{\mathrm{i}}=110 \%$ simulations, contrail depth $H$ is smaller as already observed in our EULAG-LCM simulations. The left panel reveals that the new $H$ values support the piecewise linear approximation. The right panel shows non-normalised values of $H$ and focuses on the variations in $z_{\text {desc }}$. In particular, the $H$ values of three different aircraft types in Naiman et al. (2011) (brown diamonds) are useful for comparison, as the wake vortex descent and with it the contrail depth vary over a wide range. Apparently, all $H$ values are close to the 1-to-1 line, which leads to the conclusion that the wake vortex descent is strikingly similar in all models.

\subsection{Comparison with observations}

Young contrails have been measured in situ or with lidars in the past. Unfortunately, total ice crystal number cannot reli- 
ably be determined with those methods. Lidars do not measure ice crystal numbers and in situ measurements do not sample the complete contrail, which would be necessary to derive such an integral property. Thus, we limit ourselves to compare the contrail depth with lidar measurements and ice crystal number concentrations with in situ measurements. We have seen that contrail properties depend on a multitude of parameters and usually not all of them are determined with sufficient accuracy to evaluate our proposed parametrisation. Moreover, contrails are heterogeneous and in situ measured properties may depend on how and where the contrail is sampled. As an example for the contrail heterogeneity, Fig. 14 shows the occurrence frequencies of ice crystal number concentrations inside specific 3 and 5 min old contrails. Having in mind all the above arguments, it is clear that the following exercise can only serve as general sanity check.

Freudenthaler et al. (1995) used a ground-based lidar and finds the heights of a few young contrails to range between 150 and $300 \mathrm{~m}$. We hypothesise that the contrails were produced from small to medium sized aircraft, in modestly supersaturated or stratified environments as their values do not reach our maximum values. In situ measurements of number concentrations (Voigt et al., 2011; Kaufmann et al., 2014) show mean values of around $100-200 \mathrm{~cm}^{-3}$ consistent with our data.

\section{Conclusions}

Based on the evaluation of a large data set of large-eddy simulations (LESs) of young contrails, we derived analytical approximations of contrail depth and ice crystal number for $5 \mathrm{~min}$ old contrails. At that time, the wake vortices decayed and related important aircraft-induced effects, which affect the contrail depth and crystal loss in the primary wake, ceased. The parametrisation may be implemented in contrail models that do not explicitly resolve the wake dynamics and where the contrail initialisation refers to some state after vortex break-up.

The proposed parametrisation captures the fundamental microphysical and dynamical processes in a young contrail. It takes into account the impact of ambient relative humidity $\mathrm{RH}_{\mathrm{i}}$, temperature $T_{\mathrm{CA}}$ (at cruise altitude), thermal stratification specified by the Brunt-Väisälä frequency $N_{\mathrm{BV}}$, ice crystal emission index $\mathrm{EI}_{\mathrm{iceno}}$ and aircraft parameters. The latter are the water vapour emission $\mathcal{V}$, the wing span $b$ and the initial circulation $\Gamma_{0}$ of the wake vortex. If aircraft properties are not available, empirical relationships $\mathcal{V}(b)$ and $\Gamma_{0}(b)$ are provided such that only wing span $b$ must be specified.

We find that, on average, the ice crystal number $N$ depends most sensitively on $\mathrm{EI}_{\text {iceno }}$ and $\mathrm{RH}_{\mathrm{i}}$ and to a lesser extent on $b, T_{\mathrm{CA}}$ and $N_{\mathrm{BV}}$ (in this order). For the contrail depth $H, \mathrm{RH}_{\mathrm{i}}$ and $b$ are most significant followed by $T_{\mathrm{CA}}$ and $N_{\mathrm{BV}}$. Contrail depth is independent of $\mathrm{EI}_{\text {iceno }}$ (according to the design of our parametrisation).

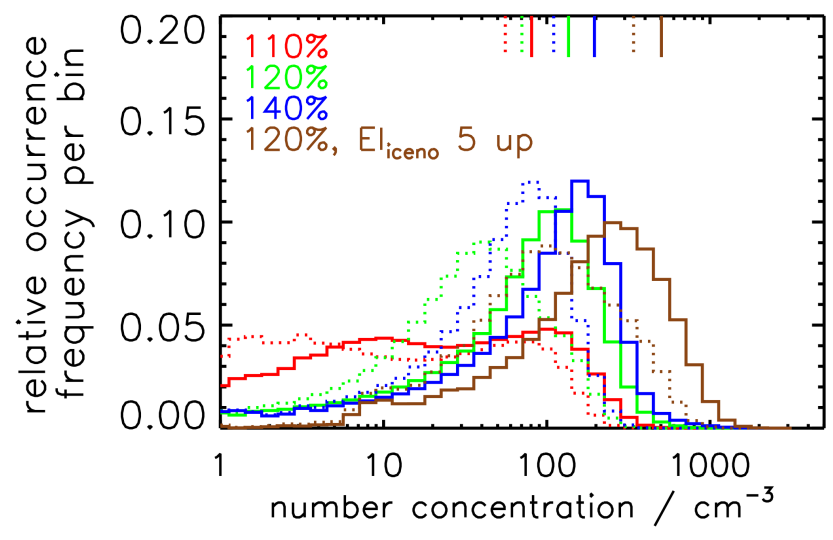

Figure 14. Relative occurrence frequencies of ice crystal number concentrations for various $\mathrm{RH}_{\mathrm{i}}$ and an elevated $\mathrm{EI}_{\mathrm{iceno}}$ (see legend). The according mean values are indicated by the vertical bars. Contrail age is $3 \mathrm{~min}$ (solid) or $5 \mathrm{~min}$ (dotted). The bin sizes increase exponentially.

For the contrail width, we recommend a value of $W=$ $150 \mathrm{~m}$. The contrail cross section is far from having a rectangular shape and the simple estimate $W \times H$ overestimates the contrail cross sectional area $A$ by up to a factor of 5. This has to be taken into account if number concentrations $n=N / A$ are derived from the parametrisation.

For young contrails evolving in ambient conditions favouring their persistence, the ranges of typical $N, H$ and $n$ values are $10^{11}-10^{13} \mathrm{~m}^{-1}, 100-600 \mathrm{~m}$ and ten to several hundred $\mathrm{cm}^{-3}$, respectively.

The parametrisation offers an ideal framework to compare simulation results from various LES models. We find an excellent agreement regarding the contrail depth and good agreement regarding the crystal number. This demonstrates the robustness of the proposed parametrisation.

A EI $I_{\text {iceno }}$ reduction from $10^{15}$ down to $10^{14} \mathrm{~kg}^{-1}$ (or $10^{14}$ down to $10^{13} \mathrm{~kg}^{-1}$ ) implies only a factor 5.3 (or 7.4) reduction of the ice crystal number in the end. An initial factor 100 reduction (from $10^{15}$ down to $10^{13} \mathrm{~kg}^{-1}$ ) gives 40 times fewer ice crystals in the end. Biofuels have lower soot emissions with a likely impact on the number of generated ice crystals. As the initial differences are reduced during the vortex phase, mitigation studies neglecting those effects may overestimate the effect of biofuels. 
Appendix A: Collection of formulae and approximations

\section{A1 Wake vortex}

The initial circulation of the wake vortices is given by

$\Gamma_{0}=\frac{g M}{\rho_{\text {air }} b_{0} U}$,

where $g$ is the gravity constant, $M$ is the aircraft mass, $U$ the cruise speed (around $230 \mathrm{~ms}^{-1}$ ) and $\rho_{\text {air }}$ the density of air (around $0.4 \mathrm{~kg} \mathrm{~m}^{-3}$ ).

The initial vortex separation $b_{0}$ is

$b_{0}=\frac{\pi}{4} b$,

where $b$ is the wing span. As the two vortices are counterrotating, one vortex has a positive sign and the other one a negative sign.

The initial descent of the wake vortex pair is given by

$w_{0}=\frac{\Gamma_{0}}{2 \pi b_{0}}$.

\section{A2 Vertical displacement}

In wake vortex research, it is common to use equations and analyse results in non-dimensional form (Gerz et al., 2002). For this, the following scaling parameters are used: length scale $b_{0}$, velocity scale $w_{0}$ and timescale $t_{0}=b_{0} / w_{0}$. Values of $w_{0}$ and $t_{0}$ for the various aircraft types are listed in Table 1.

In UG2014, the non-dimensional form was useful to find a simple parametrisation of the vertical displacement $z_{\text {desc }}^{*}=$ $z_{\text {desc }} / b_{0}$ in terms of normalised Brunt-Väisälä frequency $N_{\mathrm{BV}}^{*}=N_{\mathrm{BV}} \times t_{0}$ (see their Fig. 8 and Eq. 7). Stepping back to the dimensional form (their Eq. 8), the vertical displacement is then given by

$\hat{z}_{\mathrm{desc}}=\alpha^{\prime} b_{0}^{(1-2 \beta)} \Gamma_{0}^{\beta} N_{\mathrm{BV}}^{-\beta}$.

For the reasonable fit parameter $\beta=0.5, b_{0}$ drops out in Eq. (A4), and $\hat{z}_{\text {desc }}$ depends solely on $\Gamma_{0}$ and $N_{\mathrm{BV}}$, as given in Eq. (4).

In case, information on $\Gamma_{0}$ is not available, Sect. A3 proposes a simple relationship between $\Gamma_{0}$ and wing span.

\section{A3 Circulation estimate}

It is very conceivable that the parameter $\Gamma_{0}$ is not available in typical applications of the parametrisations. Ignoring the fact that the aircraft mass and circulation (see Eq. A1) change with time and kerosene loading, we propose the following simplification: Fig. A1a shows the relationship between wing span and initial circulation as used in UG2014. There, the initial circulation was computed assuming a medium aircraft mass (between empty aircraft and maximum takeoff mass taken from the BADA data set). Subsequently, the crude relationship

$\Gamma_{0}=(-70 \mathrm{~m}+10 b) \mathrm{ms}^{-1}$

holds, which can be plugged into Eq. (4).

Note that from a physical point of view, it is counterintuitive to integrate $b$ into an approximation of $\hat{z}_{\text {desc }}$ as it originally dropped out stepping from Eq. (A4) to Eq. (4).

\section{A4 Plume area}

For the computation of $z_{\text {emit }}$, it is important to know over which area (normal to the flight direction) the ice crystals in the primary wake are distributed. Looking at cross sections of the plume, we manually determine its radius $r_{\mathrm{p}}$ at some intermediate stage. The $r_{\mathrm{p}}$ values are depicted in Fig. A1b and are roughly linearly dependent on wing span $b$ :

$r_{\mathrm{p}}=1.5 \mathrm{~m}+0.314 b$.

The area of the two plumes is given by

$A_{\mathrm{p}}=2 \times 2 \pi r_{\mathrm{p}}^{2}$

and thus scales roughly with $b^{2}$. It is clear that once the vortices strongly oscillate, link or break up, the determination of $r_{\mathrm{p}}$ it not meaningful any longer. For each aircraft, we ran a sensitivity simulation where we increased the radius $R_{\text {init }}$ of the initial plume (Sect. 3.4 of UG2014). We find the intermediate plume radius $r_{\mathrm{p}}$ (star symbol) to be independent of the initial setting and to lie in between of the two initial values (diamond symbol).

\section{A5 Water vapour emission}

The water vapour emission is given by

$\mathcal{V}=\frac{\dot{m}_{\mathrm{f}}}{U} \mathrm{EI}_{\mathrm{H} 20}$,

UG2014 assumed a medium fuel flow representative of cruise conditions (again based on BADA data). Figure A1c suggests that $\mathcal{V}$ scales with $b^{2}$. The fit function is given by

$\mathcal{V}=0.020 \mathrm{~kg} \mathrm{~m}^{-1}(b / 80 \mathrm{~m})^{2}$

Combining Eqs. (2), (A8) and (A9) we can estimate the number of generated ice crystals by

$N_{\text {form }}=\mathrm{EI}_{\text {iceno }} \times 0.0160 \mathrm{~kg} \mathrm{~m}^{-1}(b / 80 \mathrm{~m})^{2}$.

\section{A6 Mode of use}

This section gives a summary on the presented parametrisations and deals with their computation. Note that the Supplement contains a Fortran programme for the computation of $\hat{f}_{\mathrm{Ns}}$ and $\hat{H}$. 

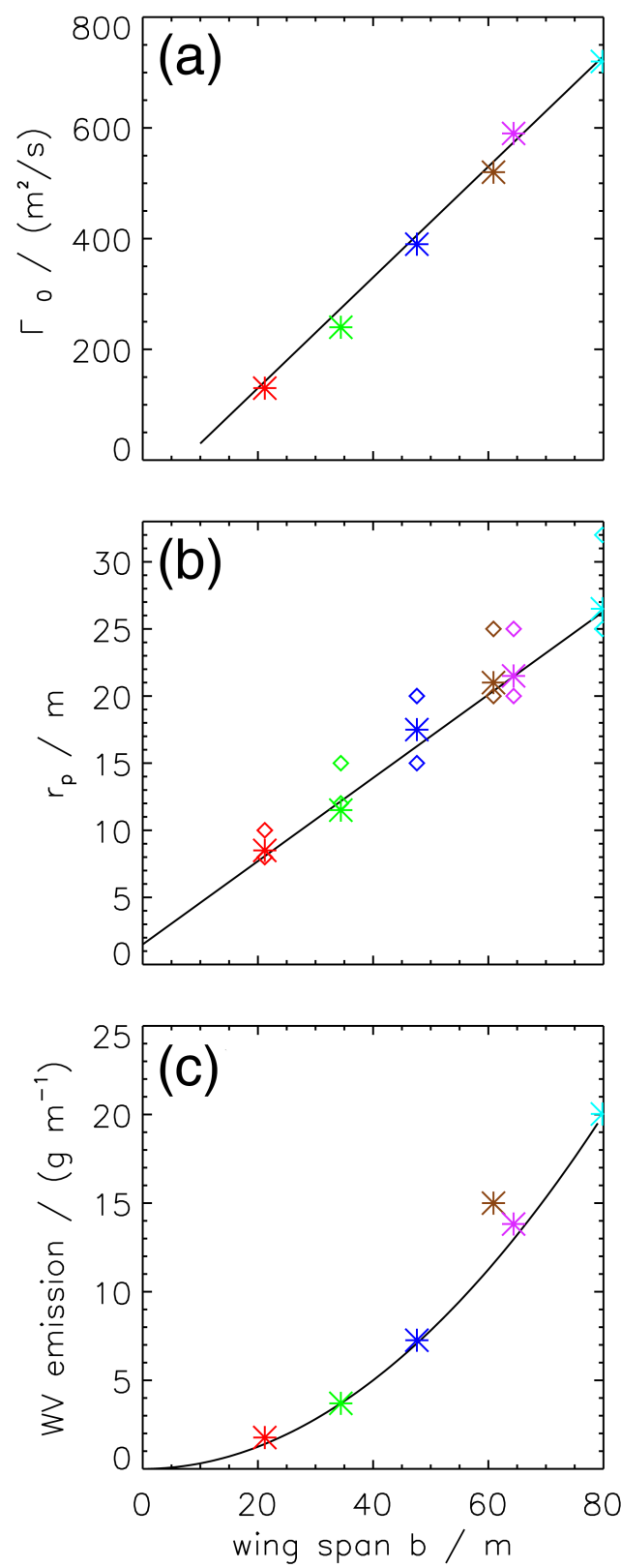

Figure A1. Top: relationship between the initial wake vortex circulation $\Gamma_{0}$ and wing span $b$ for medium aircraft mass as assumed in UG2014. The straight line shows the simple approximation $\Gamma_{0}=(-70 \mathrm{~m}+10 b) \mathrm{ms}^{-1}$. Middle: relationship between the (intermediate) plume radius $r_{\mathrm{p}}$ and $b$. The straight line shows the simple approximation $1.5 \mathrm{~m}+0.314 \mathrm{~b}$. The diamonds show two sets of initial plume radii as used in UG2014. Bottom: relationship between water vapour emission $\mathcal{V}$ and $b$ for medium fuel flow at cruise conditions as assumed in UG2014. The parabola shows the simple approximation $20 \mathrm{~g} \mathrm{~m}^{-1}(\mathrm{~b} / 80 \mathrm{~m})^{2}$.
The following input parameters have to be known:

- temperature at cruise altitude $T_{\mathrm{CA}}$

- ambient relative humidity $\mathrm{RH}_{\mathrm{i}}$

- Brunt-Väisälä frequency $N_{\mathrm{BV}}$

- wing span of the aircraft $b$

- ice crystal "emission" index $\mathrm{EI}_{\mathrm{iceno}}$

- optionally: aircraft mass

- optionally: fuel flow of the aircraft (total of all engines).

Steps for computation of $\hat{f}_{\mathrm{Ns}}$ :

1. Compute $\Gamma_{0}$ with Eq. (A1) and $\hat{z}_{\text {desc }}$ with Eq. (4). If data on the aircraft mass are not available, use the relationship given in Eq. (A5) to compute $\Gamma_{0}$.

2. Compute the concentration add-on $\rho_{\text {emit }}$ using Eqs. (6), (A7) and (A8). If data on the fuel flow are not available, use the relationship given in Eq. (A9).

3. Compute $z_{\text {atm }}$ and $z_{\text {emit }}$ via solving the non-linear Eqs. (5) and (7).

4. Compute $z_{\Delta}$ with Eq. (3) and the fit parameters given in Eqs. (10d)-(10h).

5. Compute $\hat{f}_{\mathrm{Ns}}$ with Eqs. (8) and (9) and fit parameters given in Eqs. (10a)-(10c).

6. The total ice crystal number $\hat{N}$ is given by Eq. (14). If data on the fuel flow are not available, use the relationship given in Eq. (A10) instead of Eq. (2).

Steps for computation of $\hat{H}$.

1. Steps $1-3$ as above

2. Compute $z_{\Delta}$ with Eq. (3) and the fit parameters given in Eqs. (10d)-(10f) and $\gamma_{\mathrm{atm}}=\gamma_{\mathrm{emit}}=0$.

3. Compute $\widetilde{f}_{\mathrm{Ns}}$ with Eqs. (8) and (9) and fit parameters given in Eqs. (10a)-(10c).

4. Compute $\hat{H}$ with Eqs. (12) and (13). 
Table A1. List of symbols.

\begin{tabular}{|c|c|c|}
\hline Symbol & Value/unit & Meaning \\
\hline$b$ & $\mathrm{~m}$ & wing span \\
\hline$b_{0}$ & $\mathrm{~m}$ & vortex separation \\
\hline$e_{\mathrm{V}}, e_{\mathrm{S}}$ & $\mathrm{Pa}$ & partial/saturation pressure of water vapour \\
\hline$f_{\mathrm{N}}$ & 1 & normalised ice crystal number \\
\hline$f_{\mathrm{Ns}}$ & 1 & fraction of surviving ice crystals, survival fraction \\
\hline$\hat{f}_{\mathrm{Ns}}, \widetilde{f}_{\mathrm{Ns}}$ & 1 & parametrisations of $f_{\mathrm{Ns}}$ \\
\hline $\bar{f}_{\mathrm{Ns}}$ & 1 & average survival fraction \\
\hline$\dot{m}_{\mathrm{f}}$ & $\mathrm{kg} \mathrm{s}^{-1}$ & fuel flow rate \\
\hline$\hat{n}_{\text {mean }}, n_{\text {mean }}$ & $\mathrm{m}^{-3}$ & (parametrised) ice crystal number concentration \\
\hline$r_{\mathrm{p}}$ & $\mathrm{m}$ & radius of (intermediate) plume/contrail \\
\hline$r_{\mathrm{SD}}$ & 1 & width of lognormal size distribution \\
\hline$s$ & $s^{-1}$ & vertical wind shear \\
\hline$s_{\mathrm{i}}$ & 1 & ambient supersaturation \\
\hline$x_{\mathrm{s}}$ & 1 & coefficient of the parametrisation, $>0$ \\
\hline zatm & $\mathrm{m}$ & supersaturation length scale \\
\hline$z_{\text {desc }}$ & $\mathrm{m}$ & vertical displacement of vortex system, vortex length scale \\
\hline$\hat{z}_{\text {desc }}$ & $\mathrm{m}$ & analytical approximation of $z_{\text {desc }}$ \\
\hline$z_{\text {emit }}$ & $\mathrm{m}$ & emission length scale \\
\hline$z_{\Delta}$ & $\mathrm{m}$ & balance length scale \\
\hline$A$ & $\mathrm{~m}^{2}$ & contrail area \\
\hline$A_{\mathrm{p}}$ & $\mathrm{m}^{2}$ & cross sectional area of (intermediate) plume/contrail \\
\hline $\mathrm{EI}_{\text {iceno }}$ & $(\mathrm{kg} \text { fuel })^{-1}$ & ice crystal emission index \\
\hline $\mathrm{EI}_{\text {iceno }}^{*}$ & 1 & normalised ice crystal "emission" index, $=\mathrm{EI}_{\text {iceno }} / \mathrm{EI}_{\text {iceno, ref }}$ \\
\hline $\mathrm{EI}_{\text {iceno, ref }}$ & $(\mathrm{kg} \text { fuel })^{-1}$ & reference ice crystal "emission" index, $2.8 \times 10^{14} \mathrm{~kg}^{-1}$ \\
\hline $\mathrm{EI}_{\mathrm{H}_{2} \mathrm{O}}$ & $\operatorname{kg}(\mathrm{kg} \text { fuel })^{-1}$ & water vapour "emission" index, $=1.25$ \\
\hline$H, \hat{H}$ & $\mathrm{~m}$ & simulated and parametrised contrail depth \\
\hline$I_{\mathrm{V}}$ & $\mathrm{kg} \mathrm{m}^{-2}$ & vertical profile of ice crystal mass \\
\hline$N_{\mathrm{V}}$ & $\mathrm{m}^{-2}$ & vertical profile of ice crystal number \\
\hline$N_{\mathrm{BV}}$ & $s^{-1}$ & Brunt-Väisälä frequency \\
\hline$N$ & $\mathrm{~m}^{-1}$ & ice crystal number per flight metre \\
\hline $\bar{N}_{\text {form }}, N_{\text {form }}$ & $\mathrm{m}^{-1}$ & (average) number of generated ice crystals per flight metre \\
\hline$N_{\text {soot }}$ & $m^{-1}$ & number of emitted soot particles per flight metre \\
\hline $\bar{N}_{\text {surv }}, N_{\text {surv }}$ & $\mathrm{m}^{-1}$ & (average) number of ice crystals per flight metre after vortex break-up \\
\hline$R_{\text {init }}$ & $\mathrm{m}$ & radius of initial discs with ice crystals \\
\hline$R_{\mathrm{V}}$ & $461 \mathrm{~J} \mathrm{~kg}^{-1} \mathrm{~K}^{-1}$ & gas constant of water vapour \\
\hline $\mathrm{RH}_{\mathrm{i}}$ & 1 & ambient relative humidity with respect to ice \\
\hline$T_{\mathrm{CA}}$ & $\mathrm{K}$ & temperature at cruise altitude \\
\hline$U$ & $\mathrm{~ms}^{-1}$ & cruise speed of aircraft \\
\hline$\hat{W}, W$ & $\mathrm{~m}$ & (parametrised) contrail width \\
\hline$W_{\text {rect }}$ & $\mathrm{m}$ & width of area-equivalent rectangle \\
\hline$\alpha_{\text {atm }}, \alpha_{\text {emit }}$ & 1 & coefficients of the parametrisation, $>0$ \\
\hline$\alpha_{\text {desc }}, \alpha_{0}$ & 1 & coefficients of the parametrisation, $>0$ \\
\hline$\beta_{0}, \beta_{1}$ & 1 & coefficients of the parametrisation, $>0$ \\
\hline$\eta_{1}, \eta_{2}$ & 1 & coefficients of the parametrisation, $>0$ \\
\hline$\gamma_{\mathrm{atm}}, \gamma_{\mathrm{emit}}$ & 1 & coefficients of the parametrisation, $>0$ \\
\hline$\rho_{\text {air }}$ & $\mathrm{kg} \mathrm{m}^{-3}$ & density of air \\
\hline$\rho_{\text {emit }}$ & $\mathrm{kg} \mathrm{m}^{-3}$ & emitted "concentration" \\
\hline$\Gamma_{0}$ & $\mathrm{~m}^{2} \mathrm{~s}^{-1}$ & initial circulation of vortex \\
\hline$\Gamma_{\mathrm{d}}$ & $9.8 \mathrm{~K} \mathrm{~km}^{-1}$ & dry adiabatic lapse rate \\
\hline $\mathcal{V}$ & $\mathrm{kg} \mathrm{m}^{-1}$ & water vapour emission \\
\hline
\end{tabular}


Table A2. List of simulations. Columns 2-7 list parameter settings, columns 8-11 the simulated and approximated values for survival fraction and contrail depth, and columns 12-14 the values of three characteristic length scales. "-1" indicates missing values. The aircraft (AC) type defines the wake vortex properties and water vapour emission as listed in Table 1.

\begin{tabular}{|c|c|c|c|c|c|c|c|c|c|c|c|c|c|c|}
\hline & No. & $\begin{array}{r}T_{\mathrm{CA}} \\
(\mathrm{K})\end{array}$ & $\begin{array}{r}\mathrm{RH}_{\mathrm{i}} \\
(\%)\end{array}$ & $\begin{array}{l}N_{\mathrm{BV}} \\
\left(10^{-2} \mathrm{~s}^{-1}\right)\end{array}$ & $\mathrm{AC}$ & $\begin{array}{r}\text { EI }_{\text {iceno }} \\
\left((\mathrm{kg})^{-1}\right)\end{array}$ & $\begin{array}{r}\mathcal{V} \\
\left(\mathrm{g} \mathrm{m}^{-1}\right)\end{array}$ & $f_{\mathrm{Ns}}$ & $\hat{f}_{\mathrm{Ns}}$ & $\begin{array}{r}H \\
(\mathrm{~m})\end{array}$ & $\begin{array}{r}\hat{H} \\
(\mathrm{~m})\end{array}$ & $\begin{array}{r}z_{\text {atm }} \\
(\mathrm{m})\end{array}$ & $\begin{array}{r}z_{\text {emit }} \\
(\mathrm{m})\end{array}$ & $\begin{array}{r}\hat{z}_{\text {desc }} \\
(\mathrm{m})\end{array}$ \\
\hline \multirow{18}{*}{$\begin{array}{l}\frac{\bar{v}}{0} \\
\frac{0}{n}\end{array}$} & \multicolumn{14}{|c|}{ Basic variation of $\mathrm{RH}_{\mathrm{i}}$ and $T_{\mathrm{CA}}$ for B777, taken from U2014, Fig. 4} \\
\hline & 0 & 209 & 100 & 1.15 & B777 & $2.80 \times 10^{14}$ & 15.00 & 31.2 & 38.4 & 330 & 416 & 0 & 279 & 339 \\
\hline & 1 & 209 & 120 & 1.15 & B777 & $2.80 \times 10^{14}$ & 15.00 & 89.9 & 88.2 & 420 & 441 & 137 & 279 & 339 \\
\hline & 2 & 212 & 100 & 1.15 & B777 & $2.80 \times 10^{14}$ & 15.00 & 15.8 & 14.2 & 250 & 289 & 0 & 202 & 339 \\
\hline & 3 & 212 & 120 & 1.15 & B777 & $2.80 \times 10^{14}$ & 15.00 & 81.8 & 80.5 & 420 & 437 & 141 & 202 & 339 \\
\hline & 4 & 212 & 140 & 1.15 & B777 & $2.80 \times 10^{14}$ & 15.00 & 96.5 & 93.8 & 440 & 444 & 263 & 202 & 339 \\
\hline & 5 & 217 & 100 & 1.15 & B777 & $2.80 \times 10^{14}$ & 15.00 & 3.3 & 2.8 & 110 & 57 & 0 & 117 & 339 \\
\hline & 6 & 217 & 110 & 1.15 & B777 & $2.80 \times 10^{14}$ & 15.00 & 28.1 & 21.5 & 345 & 407 & 77 & 117 & 339 \\
\hline & 7 & 217 & 120 & 1.15 & B777 & $2.80 \times 10^{14}$ & 15.00 & 65.6 & 62.5 & 420 & 428 & 148 & 117 & 339 \\
\hline & 8 & 217 & 130 & 1.15 & B777 & $2.80 \times 10^{14}$ & 15.00 & 84.6 & 83.6 & 440 & 439 & 214 & 117 & 339 \\
\hline & 9 & 217 & 140 & 1.15 & B777 & $2.80 \times 10^{14}$ & 15.00 & 92.8 & 90.9 & 450 & 443 & 276 & 117 & 339 \\
\hline & 10 & 222 & 100 & 1.15 & B777 & $2.80 \times 10^{14}$ & 15.00 & 0.3 & 0.0 & 40 & 0 & 0 & 68 & 339 \\
\hline & 11 & 222 & 110 & 1.15 & B777 & $2.80 \times 10^{14}$ & 15.00 & 16.4 & 11.6 & 180 & 235 & 81 & 68 & 339 \\
\hline & 12 & 222 & 120 & 1.15 & B777 & $2.80 \times 10^{14}$ & 15.00 & 51.8 & 47.0 & 430 & 420 & 155 & 68 & 339 \\
\hline & 13 & 222 & 140 & 1.15 & B777 & $2.80 \times 10^{14}$ & 15.00 & 89.4 & 89.1 & 460 & 442 & 289 & 68 & 339 \\
\hline & 14 & 225 & 110 & 1.15 & B777 & $2.80 \times 10^{14}$ & 15.00 & 10.8 & 9.1 & 200 & 184 & 83 & 50 & 339 \\
\hline & 15 & 225 & 120 & 1.15 & B777 & $2.80 \times 10^{14}$ & 15.00 & 44.5 & 41.7 & 450 & 418 & 160 & 50 & 339 \\
\hline & 16 & 225 & 130 & 1.15 & B777 & $2.80 \times 10^{14}$ & 15.00 & 73.0 & 76.9 & 440 & 436 & 231 & 50 & 339 \\
\hline \multirow{16}{*}{$\frac{v}{\frac{v}{0}}$} & \multicolumn{14}{|c|}{ Basic $\mathrm{RH}_{\mathrm{i}}$ variation for various aircraft types, taken from UG2014, Fig. 2} \\
\hline & 17 & 217 & 100 & 1.15 & CRJ & $2.80 \times 10^{14}$ & 1.77 & 4.7 & 9.9 & 135 & 100 & 0 & 90 & 169 \\
\hline & 18 & 217 & 100 & 1.15 & B737 & $2.80 \times 10^{14}$ & 3.70 & 3.1 & 4.9 & 80 & 68 & 0 & 83 & 230 \\
\hline & 19 & 217 & 100 & 1.15 & B767 & $2.80 \times 10^{14}$ & 7.26 & 2.0 & 2.6 & 80 & 45 & 0 & 91 & 293 \\
\hline & 20 & 217 & 100 & 1.15 & B747 & $2.80 \times 10^{14}$ & 13.82 & 4.3 & 0.5 & 120 & 11 & 0 & 98 & 361 \\
\hline & 21 & 217 & 100 & 1.15 & A380 & $2.80 \times 10^{14}$ & 20.03 & 1.6 & 0.0 & 160 & 0 & 0 & 96 & 399 \\
\hline & 22 & 217 & 120 & 1.15 & CRJ & $2.80 \times 10^{14}$ & 1.77 & 95.6 & 78.2 & 220 & 218 & 148 & 90 & 169 \\
\hline & 23 & 217 & 120 & 1.15 & B737 & $2.80 \times 10^{14}$ & 3.70 & 89.9 & 69.5 & 315 & 293 & 148 & 83 & 230 \\
\hline & 24 & 217 & 120 & 1.15 & B767 & $2.80 \times 10^{14}$ & 7.26 & 76.2 & 61.4 & 340 & 370 & 148 & 91 & 293 \\
\hline & 25 & 217 & 120 & 1.15 & B747 & $2.80 \times 10^{14}$ & 13.82 & 56.4 & 50.6 & 440 & 450 & 148 & 98 & 361 \\
\hline & 26 & 217 & 120 & 1.15 & A380 & $2.80 \times 10^{14}$ & 20.03 & 51.4 & 40.8 & 460 & 491 & 148 & 96 & 399 \\
\hline & 27 & 217 & 140 & 1.15 & CRJ & $2.80 \times 10^{14}$ & 1.77 & 99.9 & 93.6 & 220 & 222 & 276 & 90 & 169 \\
\hline & 28 & 217 & 140 & 1.15 & B737 & $2.80 \times 10^{14}$ & 3.70 & 99.4 & 92.0 & 320 & 301 & 276 & 83 & 230 \\
\hline & 29 & 217 & 140 & 1.15 & B767 & $2.80 \times 10^{14}$ & 7.26 & 96.5 & 90.8 & 370 & 383 & 276 & 91 & 293 \\
\hline & 30 & 217 & 140 & 1.15 & B747 & $2.80 \times 10^{14}$ & 13.82 & 92.2 & 89.1 & 470 & 471 & 276 & 98 & 361 \\
\hline & 31 & 217 & 140 & 1.15 & A380 & $2.80 \times 10^{14}$ & 20.03 & 89.9 & 87.4 & 490 & 519 & 276 & 96 & 399 \\
\hline \multirow{13}{*}{$\begin{array}{l}m \\
\frac{u}{0} \\
\frac{0}{n}\end{array}$} & \multicolumn{14}{|c|}{ Weaker thermal stratification, taken from UG2014, Fig. 5} \\
\hline & 32 & 217 & 140 & 0.50 & CRJ & $2.80 \times 10^{14}$ & 1.77 & 99.8 & 91.7 & 300 & 336 & 276 & 90 & 257 \\
\hline & 33 & 217 & 140 & 0.50 & B737 & $2.80 \times 10^{14}$ & 3.70 & 92.8 & 88.4 & 380 & 455 & 276 & 83 & 349 \\
\hline & 34 & 217 & 140 & 0.50 & B767 & $2.80 \times 10^{14}$ & 7.26 & 87.0 & 84.5 & 520 & 577 & 276 & 91 & 445 \\
\hline & 35 & 217 & 140 & 0.50 & B777 & $2.80 \times 10^{14}$ & 15.00 & 66.5 & 83.5 & 650 & 666 & 276 & 117 & 514 \\
\hline & 36 & 217 & 140 & 0.50 & B747 & $2.80 \times 10^{14}$ & 13.82 & 68.8 & 78.1 & 650 & 705 & 276 & 98 & 548 \\
\hline & 37 & 217 & 140 & 0.50 & A380 & $2.80 \times 10^{14}$ & 20.03 & 62.3 & 70.9 & 670 & 772 & 276 & 96 & 605 \\
\hline & 38 & 217 & 120 & 0.50 & CRJ & $2.80 \times 10^{14}$ & 1.77 & 86.7 & 67.5 & 260 & 327 & 148 & 90 & 257 \\
\hline & 39 & 217 & 120 & 0.50 & B737 & $2.80 \times 10^{14}$ & 3.70 & 63.8 & 46.5 & 400 & 433 & 148 & 83 & 349 \\
\hline & 40 & 217 & 120 & 0.50 & B767 & $2.80 \times 10^{14}$ & 7.26 & 56.1 & 29.1 & 520 & 540 & 148 & 91 & 445 \\
\hline & 41 & 217 & 120 & 0.50 & B777 & $2.80 \times 10^{14}$ & 15.00 & 31.9 & 25.8 & 620 & 622 & 148 & 117 & 514 \\
\hline & 42 & 217 & 120 & 0.50 & B747 & $2.80 \times 10^{14}$ & 13.82 & 37.0 & 15.8 & 550 & 518 & 148 & 98 & 548 \\
\hline & 43 & 217 & 120 & 0.50 & A380 & $2.80 \times 10^{14}$ & 20.03 & 29.8 & 9.6 & 550 & 350 & 148 & 96 & 605 \\
\hline
\end{tabular}


Table A2. Continued.

\begin{tabular}{|c|c|c|c|c|c|c|c|c|c|c|c|c|c|c|}
\hline & No. & $\begin{array}{r}T_{\mathrm{CA}} \\
(\mathrm{K})\end{array}$ & $\begin{array}{r}\mathrm{RH}_{\mathrm{i}} \\
(\%)\end{array}$ & $\begin{array}{l}N_{\mathrm{BV}} \\
\left(10^{-2} \mathrm{~s}^{-1}\right)\end{array}$ & $\mathrm{AC}$ & $\begin{array}{r}\mathrm{EI}_{\text {iceno }} \\
\left((\mathrm{kg})^{-1}\right)\end{array}$ & $\begin{array}{r}\mathcal{V} \\
\left(\mathrm{g} \mathrm{m}^{-1}\right)\end{array}$ & $f_{\mathrm{Ns}}$ & $\hat{f}_{\mathrm{Ns}}$ & $\begin{array}{r}H \\
(\mathrm{~m})\end{array}$ & $\begin{array}{r}\hat{H} \\
(\mathrm{~m})\end{array}$ & $\begin{array}{r}z_{\text {atm }} \\
(\mathrm{m})\end{array}$ & $\begin{array}{r}z_{\text {emit }} \\
(\mathrm{m})\end{array}$ & $\begin{array}{r}\hat{z}_{\text {desc }} \\
(\mathrm{m})\end{array}$ \\
\hline \multirow{25}{*}{$\begin{array}{l}\vec{v} \\
\frac{\ddot{U}}{m} \\
\frac{\circ}{m}\end{array}$} & \multicolumn{14}{|c|}{ Large variation of $\mathrm{EI}_{\text {iceno }}$ for B777, taken from U2014, Fig. 9 right + additional new simulations } \\
\hline & 44 & 217 & 110 & 1.15 & B777 & $1.40 \times 10^{13}$ & 15.00 & 54.1 & 78.2 & 400 & 407 & 77 & 117 & 339 \\
\hline & 45 & 217 & 120 & 1.15 & B777 & $1.40 \times 10^{13}$ & 15.00 & 94.8 & 93.3 & 420 & 428 & 148 & 117 & 339 \\
\hline & 46 & 217 & 130 & 1.15 & B777 & $1.40 \times 10^{13}$ & 15.00 & 99.8 & 97.3 & 440 & 439 & 214 & 117 & 339 \\
\hline & 47 & 217 & 110 & 1.15 & B777 & $3.22 \times 10^{13}$ & 15.00 & 45.6 & 64.3 & 380 & 407 & 77 & 117 & 339 \\
\hline & 48 & 217 & 120 & 1.15 & B777 & $3.22 \times 10^{13}$ & 15.00 & 90.3 & 89.3 & 420 & 428 & 148 & 117 & 339 \\
\hline & 49 & 217 & 130 & 1.15 & B777 & $3.22 \times 10^{13}$ & 15.00 & 98.9 & 95.3 & 440 & 439 & 214 & 117 & 339 \\
\hline & 50 & 217 & 110 & 1.15 & B777 & $5.40 \times 10^{13}$ & 15.00 & 40.9 & 52.8 & 370 & 407 & 77 & 117 & 339 \\
\hline & 51 & 217 & 120 & 1.15 & B777 & $5.40 \times 10^{13}$ & 15.00 & 86.0 & 85.6 & 420 & 428 & 148 & 117 & 339 \\
\hline & 52 & 217 & 130 & 1.15 & B777 & $5.40 \times 10^{13}$ & 15.00 & 97.6 & 93.5 & 440 & 439 & 214 & 117 & 339 \\
\hline & 53 & 217 & 110 & 1.15 & B777 & $1.27 \times 10^{14}$ & 15.00 & 34.1 & 33.8 & 360 & 407 & 77 & 117 & 339 \\
\hline & 54 & 217 & 120 & 1.15 & B777 & $1.27 \times 10^{14}$ & 15.00 & 76.5 & 76.4 & 420 & 428 & 148 & 117 & 339 \\
\hline & 55 & 217 & 130 & 1.15 & B777 & $1.27 \times 10^{14}$ & 15.00 & 93.3 & 89.5 & 440 & 439 & 214 & 117 & 339 \\
\hline & 56 & 217 & 110 & 1.15 & B777 & $6.09 \times 10^{14}$ & 15.00 & 20.7 & 14.1 & 345 & 407 & 77 & 117 & 339 \\
\hline & 57 & 217 & 120 & 1.15 & B777 & $6.09 \times 10^{14}$ & 15.00 & 51.2 & 44.7 & 420 & 428 & 148 & 117 & 339 \\
\hline & 58 & 217 & 130 & 1.15 & B777 & $6.09 \times 10^{14}$ & 15.00 & 70.1 & 74.1 & 440 & 439 & 214 & 117 & 339 \\
\hline & 59 & 217 & 110 & 1.15 & B777 & $1.40 \times 10^{15}$ & 15.00 & 14.1 & 9.2 & 345 & 407 & 77 & 117 & 339 \\
\hline & 60 & 217 & 120 & 1.15 & B777 & $1.40 \times 10^{15}$ & 15.00 & 35.5 & 28.1 & 420 & 428 & 148 & 117 & 339 \\
\hline & 61 & 217 & 130 & 1.15 & B777 & $1.40 \times 10^{15}$ & 15.00 & 50.1 & 58.1 & 440 & 439 & 214 & 117 & 339 \\
\hline & 62 & 217 & 110 & 1.15 & B777 & $3.22 \times 10^{15}$ & 15.00 & 9.0 & 6.0 & 345 & 407 & 77 & 117 & 339 \\
\hline & 63 & 217 & 120 & 1.15 & B777 & $3.22 \times 10^{15}$ & 15.00 & 22.4 & 17.6 & 420 & 428 & 148 & 117 & 339 \\
\hline & 64 & 217 & 130 & 1.15 & B777 & $3.22 \times 10^{15}$ & 15.00 & 31.7 & 38.9 & 440 & 439 & 214 & 117 & 339 \\
\hline & 65 & 217 & 110 & 1.15 & B777 & $7.00 \times 10^{15}$ & 15.00 & 5.5 & 3.9 & 345 & 407 & 77 & 117 & 339 \\
\hline & 66 & 217 & 120 & 1.15 & B777 & $7.00 \times 10^{15}$ & 15.00 & 19.5 & 11.6 & 420 & 428 & 148 & 117 & 339 \\
\hline & 67 & 217 & 130 & 1.15 & B777 & $7.00 \times 10^{15}$ & 15.00 & 19.5 & 25.0 & 440 & 439 & 214 & 117 & 339 \\
\hline \multirow{5}{*}{$\frac{n}{\frac{n}{0}}$} & \multicolumn{14}{|c|}{ Variation of fuel flow for B777, taken from U2014, Fig. 9 middle } \\
\hline & 68 & 217 & 110 & 1.15 & B777 & $2.80 \times 10^{14}$ & 12.00 & 22.8 & 15.8 & 320 & 322 & 77 & 95 & 339 \\
\hline & 69 & 217 & 110 & 1.15 & B777 & $2.80 \times 10^{14}$ & 18.00 & 31.6 & 28.6 & 370 & 411 & 77 & 139 & 339 \\
\hline & 70 & 217 & 120 & 1.15 & B777 & $2.80 \times 10^{14}$ & 12.00 & 64.7 & 54.0 & 420 & 424 & 148 & 95 & 339 \\
\hline & 71 & 217 & 120 & 1.15 & B777 & $2.80 \times 10^{14}$ & 18.00 & 67.0 & 69.4 & 420 & 432 & 148 & 139 & 339 \\
\hline \multirow{11}{*}{$\begin{array}{l}0 \\
\text { 음 } \\
\frac{0}{n}\end{array}$} & \multicolumn{14}{|c|}{ Small variation of $\mathrm{EI}_{\text {iceno }}$ for all AC, taken from UG2014, Fig. 6} \\
\hline & 72 & 217 & 120 & 1.15 & CRJ & $6.44 \times 10^{13}$ & 1.77 & 100.0 & 88.9 & 220 & 218 & 148 & 90 & 169 \\
\hline & 73 & 217 & 120 & 1.15 & B737 & $6.44 \times 10^{13}$ & 3.70 & 98.5 & 85.4 & 315 & 293 & 148 & 83 & 230 \\
\hline & 74 & 217 & 120 & 1.15 & B767 & $6.44 \times 10^{13}$ & 7.26 & 92.4 & 82.8 & 340 & 370 & 148 & 91 & 293 \\
\hline & 75 & 217 & 120 & 1.15 & B747 & $6.44 \times 10^{13}$ & 13.82 & 75.6 & 79.1 & 440 & 450 & 148 & 98 & 361 \\
\hline & 76 & 217 & 120 & 1.15 & A 380 & $6.44 \times 10^{13}$ & 20.03 & 70.0 & 74.6 & 460 & 491 & 148 & 96 & 399 \\
\hline & 77 & 217 & 120 & 1.15 & CRJ & $1.22 \times 10^{15}$ & 1.77 & 70.1 & 58.4 & 220 & 218 & 148 & 90 & 169 \\
\hline & 78 & 217 & 120 & 1.15 & B737 & $1.22 \times 10^{15}$ & 3.70 & 59.9 & 42.9 & 315 & 293 & 148 & 83 & 230 \\
\hline & 79 & 217 & 120 & 1.15 & B767 & $1.22 \times 10^{15}$ & 7.26 & 47.4 & 31.6 & 340 & 370 & 148 & 91 & 293 \\
\hline & 80 & 217 & 120 & 1.15 & B747 & $1.22 \times 10^{15}$ & 13.82 & 32.7 & 21.8 & 440 & 450 & 148 & 98 & 361 \\
\hline & 81 & 217 & 120 & 1.15 & A380 & $1.22 \times 10^{15}$ & 20.03 & 29.5 & 16.1 & 460 & 491 & 148 & 96 & 399 \\
\hline \multirow{28}{*}{$\begin{array}{l}\frac{y}{0} \\
\frac{o}{n}\end{array}$} & & & & n Lewel & 1. $(2014$ & Figs. 2, 9 and & $H$ valu & from & Zuebsc & and 1 & well & 2006 & ig. 6) & \\
\hline & 82 & 205 & 110 & 0.88 & B767 & $1.00 \times 10^{15}$ & 7.26 & 77.0 & 67.4 & -1 & 435 & 69 & 339 & 335 \\
\hline & 83 & 205 & 130 & 0.88 & B767 & $1.00 \times 10^{15}$ & 7.26 & 95.0 & 89.1 & -1 & 440 & 191 & 339 & 335 \\
\hline & 84 & 218 & 110 & 0.86 & B767 & $1.00 \times 10^{15}$ & 7.26 & 24.9 & 6.8 & 200 & 266 & 78 & 81 & 339 \\
\hline & 85 & 218 & 130 & 0.86 & B767 & $1.00 \times 10^{15}$ & 7.26 & 48.0 & 55.6 & 470 & 437 & 216 & 81 & 339 \\
\hline & 86 & 225 & 110 & 0.84 & B767 & $1.00 \times 10^{15}$ & 7.26 & 12.0 & 3.5 & -1 & 147 & 83 & 38 & 343 \\
\hline & 87 & 225 & 130 & 0.84 & B767 & $1.00 \times 10^{15}$ & 7.26 & 35.0 & 47.3 & -1 & 440 & 231 & 38 & 343 \\
\hline & 88 & 218 & 110 & 0.86 & B767 & $1.00 \times 10^{17}$ & 7.26 & 0.5 & 0.0 & -1 & 266 & 78 & 81 & 339 \\
\hline & 89 & 218 & 110 & 0.86 & B767 & $1.00 \times 10^{16}$ & 7.26 & 4.6 & 1.7 & -1 & 266 & 78 & 81 & 339 \\
\hline & 90 & 218 & 110 & 0.86 & B767 & $1.00 \times 10^{14}$ & 7.26 & 49.3 & 22.9 & -1 & 266 & 78 & 81 & 339 \\
\hline & 91 & 218 & 110 & 0.86 & B767 & $1.00 \times 10^{13}$ & 7.26 & 72.9 & 69.2 & -1 & 266 & 78 & 81 & 339 \\
\hline & 92 & 218 & 110 & 0.86 & B767 & $1.00 \times 10^{12}$ & 7.26 & 95.6 & 91.8 & -1 & 266 & 78 & 81 & 339 \\
\hline & 93 & 218 & 110 & 0.86 & B767 & $1.00 \times 10^{11}$ & 7.26 & 100.0 & 98.4 & -1 & 266 & 78 & 81 & 339 \\
\hline & & & & & $H$ value & from Naiman & al. (2011 & Figs. 1 & $, 11,13$ & and 14 & & & & \\
\hline & 94 & 219 & 130 & 1.00 & B767N & $1.00 \times 10^{15}$ & 7.25 & 25.0 & 59.2 & 400 & 407 & 218 & 74 & 315 \\
\hline & 95 & 219 & 130 & 1.00 & B747N & $1.00 \times 10^{15}$ & 14.50 & 18.0 & 42.5 & 520 & 518 & 218 & 83 & 405 \\
\hline & 96 & 219 & 130 & 1.00 & B737N & $1.00 \times 10^{15}$ & 3.13 & 35.0 & 66.3 & 320 & 323 & 218 & 57 & 250 \\
\hline & 97 & 219 & 120 & 1.00 & B767N & $1.00 \times 10^{15}$ & 7.25 & 23.0 & 26.9 & -1 & 393 & 151 & 74 & 315 \\
\hline & 98 & 219 & 110 & 1.00 & B767N & $1.00 \times 10^{15}$ & 7.25 & 21.0 & 7.8 & -1 & 271 & 78 & 74 & 315 \\
\hline & 99 & 219 & 130 & 1.00 & B767N & $1.00 \times 10^{14}$ & 7.25 & 91.0 & 88.7 & -1 & 407 & 218 & 74 & 315 \\
\hline & 100 & 219 & 110 & 1.00 & B767N & $1.00 \times 10^{14}$ & 7.25 & 88.0 & 24.5 & -1 & 271 & 78 & 74 & 315 \\
\hline & 101 & 219 & 130 & 1.50 & B767N & $1.00 \times 10^{15}$ & 7.25 & 25.0 & 69.1 & 300 & 333 & 218 & 74 & 257 \\
\hline & & & & & $H \vee$ & lue from Paug & et al. (2 & 10, Fig & & & & & & \\
\hline & 102 & 220 & 130 & 1.40 & B747P1 & $1.50 \times 10^{14}$ & 12.50 & -1.0 & 85.5 & 430 & 425 & 220 & 73 & 330 \\
\hline & & s & 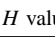 & s from $\mathrm{Pic}$ & 2015 & Table 3 Fig & 20 & 年 & (2) & & 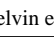 & 年 & 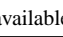 & \\
\hline & 103 & 218 & 130 & 1.20 & B747P2 & $8.30 \times 10^{13}$ & 15.00 & 98.0 & 91.2 & 440 & 447 & 216 & 108 & 346 \\
\hline & 104 & 218 & 110 & 1.20 & $\mathrm{~B} 747 \mathrm{P} 2$ & $8.30 \times 10^{13}$ & 15.00 & 65.0 & 37.1 & 380 & 382 & 78 & 108 & 346 \\
\hline & 105 & 215 & 130 & 1.20 & B747P2 & $8.30 \times 10^{13}$ & 15.00 & 99.0 & 93.0 & -1 & 449 & 210 & 150 & 346 \\
\hline
\end{tabular}




\section{The Supplement related to this article is available online at doi:10.5194/acp-16-2059-2016-supplement.}

Acknowledgements. The author is partly funded by the DFG (German Science Foundation, contract number UN286/1-2). Computational resources were made available by the German Climate Computing Center (DKRZ) through support from the German Federal Ministry of Education and Research (BMBF). I thank my former student N. Görsch for preparatory work and several DLR colleagues for discussions. I am indebted to D. Lewellen and R. Paoli who provided me with their simulation data.

The article processing charges for this open-access

publication were covered by a Research

Centre of the Helmholtz Association.

Edited by: B. Vogel

\section{References}

Boucher, O., Randall, D., Artaxo, P., Bretherton, C., Feingold, G., Forster, P., Kerminen, V.-M., Kondo, Y., Liao, H., Lohmann, U., Rasch, P., Satheesh, S., Sherwood, S., Stevens, B., and Zhang, X.: Clouds and Aerosols, book section 7, 571-658, Cambridge University Press, Cambridge, UK and NY, USA, doi:10.1017/CBO9781107415324.016, 2013.

Burkhardt, U. and Kärcher, B.: Process-based simulation of contrail cirrus in a global climate model, J. Geophys. Res., 114, D16201, doi:10.1029/2008JD011491, 2009.

Burkhardt, U. and Kärcher, B.: Global radiative forcing from contrail cirrus, Nature Climate Change, 1, 54-58, 2011.

Chen, C.-C. and Gettelman, A.: Simulated radiative forcing from contrails and contrail cirrus, Atmos. Chem. Phys., 13, 1252512536, doi:10.5194/acp-13-12525-2013, 2013.

Crow, S.: Stability theory for a pair of trailing vortices, AIAA J., 8, 2172-2179, 1970.

Freudenthaler, V., Homburg, F., and Jäger, H.: Contrail observations by ground-based scanning lidar: Cross-sectional growth, Geophys. Res. Lett., 22, 3501-3504, 1995.

Garnier, F., Maglaras, E., Morency, F., and Vancassel, X.: Effect of Compressibility on Contrail Ice Particle Growth in an Engine Jet, Int. J. Turbo. Jet. Eng., 31, 131-140, 2014.

Gerz, T., Holzäpfel, F., and Darracq, D.: Commercial aircraft wake vortices, Prog. Aerosp. Sci., 38, 181-208, 2002.

Ghosh, S., Dobbie, S., Marsham, J., and Jonas, P.: On the importance of the diffusional uptake of water vapour for the development and radiative properties of high altitude clouds: a large eddy model sensitivity study, Q. J. Roy. Meteor. Soc., 133, 1731-1741, 2007.

Hennemann, I. and Holzäpfel, F.: Large-eddy simulation of aircraft wake vortex deformation and topology, P. I. Mech. Eng. G.-J. Aer., 225, 1336-1350, doi:10.1177/0954410011402257, 2011.
Hoshizaki, H., Anderson, L. B., Conti, R. J., Farlow, N., Meyer, J. W., Overcamp, T., Redler, K. O., and Watson, V.: Aircraft Wake Microscale Phenomena, in: The Stratosphere perturbed by Propulsion Effluents, U. S. Dep. of Transportation DOT-TST-7553, Washington, D.C., USA, 1975.

Huebsch, W. and Lewellen, D.: Sensitivity Study on Contrail Evolution, 36th AIAA Fluid Dynamics Conference and Exhibit 5-8 June 2006, San Francisco, USA, AIAA 2006-3749, 1-14, doi:10.2514/6.2006-3749, 2006.

Kärcher, B. and Yu, F.: Role of aircraft soot emissions in contrail formation, Geophys. Res. Lett., 36, L01804, doi:10.1029/2008GL036649, 2009.

Kärcher, B., Busen, R., Petzold, A., Schröder, F. P., Schumann, U., and Jensen, E. J.: Physicochemistry of aircraft-generated liquid aerosols, soot, and ice particles. 2. Comparison with observations and sensitivity studies, J. Geophys. Res., 103, 1712917148, 1998.

Kärcher, B., Burkhardt, U., Unterstrasser, S., and Minnis, P.: Factors controlling contrail cirrus optical depth, Atmos. Chem. Phys., 9 , 6229-6254, doi:10.5194/acp-9-6229-2009, 2009.

Kaufmann, S., Voigt, C., Jeßberger, P., Jurkat, T., Schlager, H., Schwarzenboeck, A., Klingebiel, M., and Thornberry, T.: In situ measurements of ice saturation in young contrails, Geophys. Res. Lett., 41, 702-709, doi:10.1002/2013GL058276, 2014.

Lewellen, D. and Lewellen, W.: The effects of aircraft wake dynamics on contrail development, J. Atmos. Sci., 58, 390-406, 2001.

Lewellen, D., Lewellen, W., Poole, L., DeCoursey, R., Hansen, G., Hostetler, C., and Kent, G.: Large-eddy simulations and lidar measurements of vortex-pair breakup in aircraft wakes, AIAA J., 36, 1439-1445, 1998.

Lewellen, D. C.: Persistent contrails and contrail cirrus. Part 2: Full Lifetime Behavior, J. Atmos. Sci., 71, 4420-4438, doi:10.1175/JAS-D-13-0317.1, 2014.

Lewellen, D. C., Meza, O., and Huebsch, W. W.: Persistent contrails and contrail cirrus. Part 1: Large-eddy simulations from inception to demise, J. Atmos. Sci., 71, 4399-4419, doi:10.1175/JASD-13-0316.1, 2014.

Naiman, A. D., Lele, S. K., and Jacobsen, M. Z.: Large eddy simulations of contrail development: Sensitivity to initial and ambient conditions over first twenty minutes, J. Geophys. Res., 116, D21208, doi:10.1029/2011JD015806, 2011.

Nuic, A.: User manual for the Base of Aircraft Data (BADA), Revision 3.9, Tech. rep., EEC Technical/Scientific Report No. 11/03/08-08, EUROCONTROL, Bruxelles, Belgium, 2011.

Paoli, R., Hélie, J., and Poinsot, T.: Contrail formation in aircraft wakes, J. Fluid Mech., 502, 361-373, 2004.

Paoli, R., Nybelen, L., Picot, J., and Cariolle, D.: Effects of jet/vortex interaction on contrail formation in supersaturated conditions, Phys. Fluids, 25, 1-28, 2013.

Paugam, R., Paoli, R., and Cariolle, D.: Influence of vortex dynamics and atmospheric turbulence on the early evolution of a contrail, Atmos. Chem. Phys., 10, 3933-3952, doi:10.5194/acp-103933-2010, 2010.

Picot, J., Paoli, R., Thouron, O., and Cariolle, D.: Large-eddy simulation of contrail evolution in the vortex phase and its interaction with atmospheric turbulence, Atmos. Chem. Phys., 15, 73697389, doi:10.5194/acp-15-7369-2015, 2015. 
Ponater, M., Marquart, S., and Sausen, R.: Contrails in a comprehensive global climate model: Parameterization and radiative forcing results, J. Geophys. Res., 107, 941-960, 2002.

Pruppacher, H. and Klett, J.: Microphysics of clouds and precipitation, Kluwer Academic Publishers, Dordrecht, the Netherlands, 1997.

Rap, A., Forster, P., Jones, A., Boucher, O., Haywood, J., Bellouin, N., and De Leon, R.: Parameterization of contrails in the UK Met Office Climate Model, J. Geophys. Res., 115, D10205, doi:10.1029/2009JD012443, 2010.

Schumann, U.: On conditions for contrail formation from aircraft exhausts, Meteorol. Z., 5, 4-23, 1996.

Schumann, U.: A contrail cirrus prediction model, Geosci. Model Dev., 5, 543-580, doi:10.5194/gmd-5-543-2012, 2012.

Sölch, I. and Kärcher, B.: A large-eddy model for cirrus clouds with explicit aerosol and ice microphysics and Lagrangian ice particle tracking, Q. J. Roy. Meteor. Soc., 136, 2074-2093, 2010.

Sussmann, R. and Gierens, K.: Lidar and numerical studies on the different evolution of vortex pair and secondary wake in young contrails, J. Geophys. Res., 104, 2131-2142, 1999.

Unterstraßer, S.: Numerische Simulationen von Kondensstreifen und deren Übergang in Zirren, PhD thesis, LMU München, Germany, 2008.

Unterstrasser, S.: Large eddy simulation study of contrail microphysics and geometry during the vortex phase and consequences on contrail-to-cirrus transition, J. Geophys. Res., 119, 75377555, doi:10.1002/2013JD021418, 2014.

Unterstrasser, S. and Gierens, K.: Numerical simulations of contrail-to-cirrus transition - Part 1: An extensive parametric study, Atmos. Chem. Phys., 10, 2017-2036, doi:10.5194/acp-102017-2010, 2010a.
Unterstrasser, S. and Gierens, K.: Numerical simulations of contrail-to-cirrus transition - Part 2: Impact of initial ice crystal number, radiation, stratification, secondary nucleation and layer depth, Atmos. Chem. Phys., 10, 2037-2051, doi:10.5194/acp-102037-2010, 2010b.

Unterstrasser, S. and Görsch, N.: Aircraft-type dependency of contrail evolution, J. Geophys. Res., 119, 14015-14027, doi:10.1002/2014JD022642, 2014.

Unterstrasser, S. and Sölch, I.: Study of contrail microphysics in the vortex phase with a Lagrangian particle tracking model, Atmos. Chem. Phys., 10, 10003-10015, doi:10.5194/acp-1010003-2010, 2010.

Unterstrasser, S., Gierens, K., and Spichtinger, P.: The evolution of contrail microphysics in the vortex phase, Meteorol. Z., 17, 145156, 2008.

Unterstrasser, S., Paoli, R., Sölch, I., Kühnlein, C., and Gerz, T.: Dimension of aircraft exhaust plumes at cruise conditions: effect of wake vortices, Atmos. Chem. Phys., 14, 2713-2733, doi:10.5194/acp-14-2713-2014, 2014.

Voigt, C., Schumann, U., Jessberger, P., Jurkat, T., Petzold, A., Gayet, J.-F., Krämer, M., Thornberry, T., and Fahey, D.: Extinction and optical depth of contrails, Geophys. Res. Lett., 38, L11806, doi:10.1029/2011GL047189, 2011.

Yu, F. and Turco, R. P.: Contrail formation and impacts on aerosol properties in aircraft plumes: Effects of fuel sulfur content, Geophys. Res. Lett., 25, 313-316, 1998. 